\title{
ON THE YAU CYCLE OF A NORMAL SURFACE SINGULARITY*
}

\author{
KAZUHIRO KONNO ${ }^{\dagger}$
}

\begin{abstract}
The notion of the Yau sequence was introduced by Tomaru, as an attempt to extend Yau's elliptic sequence for (weakly) elliptic singularities to normal surface singularities of higher fundamental genera. We show some fundamental properties of the sequence. Among other things, it is shown that its length gives us the arithmetic genus for singular points of fundamental genus two. Furthermore, an upper bound on the geometric genus is given for certain surface singularities of degree one. The relation between the canonical cycle and the Yau cycle is also discussed.
\end{abstract}

Key words. Surface singularity, Yau cycle, canonical cycle.

AMS subject classifications. $14 \mathrm{~J} 17$.

Introduction. Let $(V, o)$ be a germ of a normal singular point on a complex surface $V$. If $\pi: X \rightarrow V$ denotes a resolution, then the intersection form is negative definite on the exceptional set $\pi^{-1}(o)$. Hence, there exists a non-zero effective divisor with support $\pi^{-1}(o)$ that has a non-positive intersection number with every exceptional curve. We denote by $Z$ the smallest one among such divisors and call it the fundamental cycle. Apparently, $-Z^{2}$ is one of the most naive invariants of $(V, o)$ independent of the choice of resolutions. We call $-Z^{2}$ the degree of $(V, o)$.

In this paper, we study surface singularities by considering decompositions of various cycles on $\pi^{-1}(o)$. One of the main objects is the Yau sequence introduced by Tomaru [9], which formally generalizes S.S.T. Yau's elliptic sequence [11] to singularities of bigger fundamental genera. We define the Yau cycle $Y$ to be the sum of all curves appearing in the sequence. Then one can associate to $(V, o)$ some new numerical invariants such as $-Y^{2}, p_{a}(Y)$ and $\operatorname{dim} H^{1}\left(Y, \mathcal{O}_{Y}\right)$. Furthermore, as is naturally expected after [11], $Y$ enjoys nice numerical properties similar to those of the canonical cycle of a numerically Gorenstein elliptic singularity. Though, in this paper, we can only give small applications with a special regard to singularities of degree one, we hope that the Yau cycle will work in large for fruitful results in future studies of surface singularities of general type.

The organization of the paper is as follows. In $\S 1$, we recall the notion of the Yau sequence [9] and state fundamental properties of cycles canonically associated to the sequence. Several known facts on the elliptic sequence (see, e.g., [11], [7], [5]) will be successfully extended to Yau cycles. Among other things, in Theorem 1.5, we give a formula computing $\operatorname{dim} H^{1}\left(Y, \mathcal{O}_{Y}\right)$ in the spirit of $[6]$. In $\S 2$, we observe the relation between Yau sequence and the arithmetic genus of a singular point of fundamental genus 2, and show in Corollary 2.5 that the length of the sequence actually computes the arithmetic genus. We also discuss two conjectures posed by Okuma in [6] for numerically Gorenstein elliptic singularities. We give an affirmative answer to Conjecture 1.4 and a counterexample to Conjecture 5.14 in [6].

The rest is basically devoted to singularities of degree one. Such singular points are attractive not only for the naive reason that the degree is the smallest possible, but also for the fact shown in [2] that each connected component of the base locus of the linear system $|L|$ is contained in the exceptional set of a singularity of degree one,

\footnotetext{
*Received January 20, 2010; accepted for publication July 20, 2011.

$\dagger$ Department of Mathematics, Graduate School of Science, Osaka University, Toyonaka, Osaka 560-0043, Japan (konno@math.sci.osaka-u.ac.jp).
} 
for any invertible sheaf $L$ on $X$ such that $L-K_{X}$ is nef. In $\S 3$, we study the Yau cycle on the minimal resolution and show in Lemma 3.4 that some multiple of it gives us the canonical cycle, when $Z$ is essentially irreducible, a condition automatically satisfied in the elliptic case. See Theorem 3.5 for a slightly more general result that gives a sufficient condition for a singularity of degree one to be numerically Gorenstein and shows how Yau cycles can describe the canonical cycle. For those with essentially irreducible $Z$, we also give in Theorem 3.9 the upper bound of the geometric genus. Example 3.10 tells us that the bound is optimal. In $\S 4$, we discuss decompositions of the canonical cycle of a numerically Gorenstein singular point in order to supplement a result in [3]. Theorem 4.5 shows, as predicted by Theorem 3.5, that a certain multiple of the Yau cycle forms the "leading term" of the canonical cycle when the singular point is of degree one. Theorem 4.7 describes the case of fundamental genus 2 (of given degree).

Notation. Throughout the paper, a curve means a non-zero effective divisor (with compact irreducible components) on a non-singular complex surface. For a curve $D$, the arithmetic genus $p_{a}(D)$ is defined by $p_{a}(D)=1-\chi\left(\mathcal{O}_{D}\right)$. If $D$ is on a non-singular surface $X$, then the invertible sheaf $\mathcal{O}_{D}\left(K_{X}+D\right)$ is the dualizing sheaf $\omega_{D}$ of $D$, by the adjunction formula. We have $2 p_{a}(D)-2=\operatorname{deg} \omega_{D}=D\left(K_{X}+D\right)$. An invertible sheaf (or a line bundle) on a curve is nef if it is of non-negative degree on any irreducible components.

A curve $D$ is chain-connected if $\mathcal{O}_{D-\Gamma}(-\Gamma)$ is not nef for any proper subcurve $\Gamma$, $0 \prec \Gamma \prec D$. One of the remarkable features of a chain-connected curve $D$ is that, if $\mathcal{O}_{D}(-C)$ is nef for a curve $C$, then either $D \preceq C$ or $\operatorname{Supp}(C) \cap \operatorname{Supp}(D)=\emptyset$. If $D$ is chain-connected and $p_{a}(D)>0$, then there uniquely exists a chain-connected subcurve $D_{\min }$ of $D$ such that $p_{a}\left(D_{\min }\right)=p_{a}(D)$ and $K_{D_{\min }}$ is nef. We call $D_{\min }$ the minimal model of $D$. We have

$$
\begin{aligned}
D_{\min } & =\min \left\{\Gamma \mid 0 \prec \Gamma \preceq D, p_{a}(\Gamma)=p_{a}(D)\right\} \\
& =\max \left\{\Gamma \mid 0 \prec \Gamma \preceq D, K_{\Gamma} \text { is nef }\right\} .
\end{aligned}
$$

A maximal chain-connected subcurve of a curve $C$ is called a chain-connected component of $C$. Every curve $C$ decomposes as $C=\sum_{i=1}^{n} C_{i}$ in such a way that $C_{i}$ is a chain-connected component of $C-\sum_{j<i} C_{j}$. Then $\mathcal{O}_{C_{j}}\left(-C_{i}\right)$ is nef for $i<j$. Such an ordered decomposition is essentially unique and called a chain-connected component decomposition (a CCC decomposition, for short) of $C$. See [3] for these facts and further properties.

We sometimes need a stronger connectivity for curves. For a fixed integer $k$ (usually non-negative), $D$ is called (numerically) $k$-connected, if $(D-\Gamma) \Gamma \geq k$ holds for any proper subcurve $\Gamma \prec D$. Every 1-connected curve is chain-connected. But the converse is not true. For further properties of numerically connected curves, we refer the readers to [1, Appendix].

Let $(V, o)$ be an isolated surface singularity and $\pi: X \rightarrow V$ a resolution. Let $Z$ be the fundamental cycle on $\pi^{-1}(o)$. Then $Z$ is chain-connected. We call the arithmetic genus of $Z$ the fundamental genus of $(V, o)$ and denote it by $p_{f}(V, o)$. The arithmetic genus and the geometric genus of $(V, o)$ are respectively defined by $p_{a}(V, o)=\max \left\{p_{a}(D) \mid 0 \prec D, \operatorname{Supp}(D) \subseteq \pi^{-1}(o)\right\}$ and $p_{g}(V, o)=\operatorname{dim}\left(R^{1} \pi_{*} \mathcal{O}_{X}\right)_{o}$. It is known that $p_{f}(V, o) \leq p_{a}(V, o) \leq p_{g}(V, o)$. See [10]. Since the intersection form is negative definite on $\pi^{-1}(o)$, there is a $\mathbb{Q}$-divisor $Z_{K}$ with support in $\pi^{-1}(o)$ such that $-Z_{K}$ is numerically equivalent to $K_{X}$. We call it the canonical cycle. When $Z_{K}$ is 
an integral divisor, we call $(V, o)$ a numerically Gorenstein singularity. Note that a normal surface singularity $(V, o)$ is Gorenstein i.e., $\mathcal{O}_{V, o}$ is a Gorenstein local ring, if and only if $-Z_{K}$ is linearly equivalent to $K_{X}$.

Acknowledgments. The author would like to thank Tadashi Tomaru very much for his interests and helpful suggestions.

1. The minimal model and Yau cycle. In this section, we recall the notion of the Yau sequence introduced by Tomaru [9] and study cycles canonically associated to it.

Let $(V, o)$ be a germ of a normal surface singular point and $\pi: X \rightarrow V$ a resolution. We denote by $Z$ the fundamental cycle on $\pi^{-1}(o)$ and always assume that $p_{f}(V, o):=$ $p_{a}(Z)>0$. Let $Z_{\min }$ denote the minimal model of $Z$. Recall that $Z_{\min }$ is obtained from $Z$ by removing $(-1)_{Z}$-curves, i.e., non-singular rational curves $C$ with $C(Z-C)=1$, one by one (see, [3, Sect.2]). Note also that $Z-C$ is chain-connected, $H^{1}\left(Z, \mathcal{O}_{Z}\right) \simeq$ $H^{1}\left(Z-C, \mathcal{O}_{Z-C}\right)$ and $H^{1}(Z, \mathbb{Z}) \simeq H^{1}(Z-C, \mathbb{Z})$ for any $(-1)_{Z}$-curve $C$ (if exists).

LEMMA 1.1. Assume that $-Z$ is numerically trivial on $Z_{\min }$. Let $D \prec Z$ be a maximal subcurve such that $\mathcal{O}_{D}(-Z)$ is numerically trivial and $p_{a}(D)=p_{f}(V, o)$. Then the following hold.

(1) $D$ is the fundamental cycle on its support.

(2) $\Delta \preceq D$ holds for any chain-connected curve $\Delta \preceq Z$ such that $\mathcal{O}_{\Delta}(-Z)$ is numerically trivial and $p_{a}(\Delta)>0$.

(3) If $C \prec Z$ is an irreducible component such that $C D>0$, then $C \simeq \mathbb{P}^{1}, C D=1$ and $C Z<0$.

Proof. Let $D$ be a maximal subcurve of $Z$ such that $\mathcal{O}_{D}(-Z)$ is numerically trivial and $p_{a}(D)=p_{f}(V, o)$. Recall that the last condition assures the chain-connectivity of $D$ by [3, Lemma 3.2]. We show (1) holds for $D$. Assume that there is a component $C \preceq D$ satisfying $C D>0$. Since $\mathcal{O}_{D}(-Z)$ is numerically trivial and $C \preceq D$, we have $C(Z-D)<0$ and, hence, $C \preceq Z-D$. Then $C+D$ is a subcurve of $Z$ and $\mathcal{O}_{C+D}(-Z)$ is numerically trivial. Furthermore, we have $p_{a}(D+C)=p_{a}(D)+p_{a}(C)-$ $1+C D \geq p_{a}(D)=p_{f}$. Then $p_{a}(D+C)=p_{f}$, since $p_{a}(D+C) \leq h^{1}\left(D+C, \mathcal{O}_{D+C}\right) \leq$ $h^{1}\left(Z, \mathcal{O}_{Z}\right)=p_{f}$. This contradicts the assumption that $D$ is maximal. Hence we get (1).

Let $\Delta$ be as in (2). If $\mathcal{O}_{\Delta}(-D)$ is not nef, then there exists a component $C \preceq \Delta$ satisfying $C D>0$. Since $D$ is the fundamental cycle on its support, this shows $C \npreceq D$ and it follows $C+D \preceq Z$. But, $\mathcal{O}_{C+D}(-Z)$ is numerically trivial and $p_{a}(D+C)=p_{f}$, contradicting the maximality of $D$. Hence $\mathcal{O}_{\Delta}(-D)$ is nef. Then, since $\Delta$ is chainconnected, either $\operatorname{Supp}(\Delta) \cap \operatorname{Supp}(D)=\emptyset$ or $\Delta \preceq D$. If the first alternative happens, then $D+\Delta \preceq Z$ and we would have $p_{a}(D)+p_{a}(\Delta)=h^{1}\left(D+\Delta, \mathcal{O}_{D+\Delta}\right) \leq h^{1}\left(Z, \mathcal{O}_{Z}\right)=$ $p_{f}$, which is impossible by $p_{a}(D)=p_{f}$ and $p_{a}(\Delta)>0$. Therefore, $\Delta$ is a subcurve of $D$.

We show (3). Since we must have $p_{a}(D+C)=p_{a}(D)$, we get $p_{a}(C)-1+C D=0$. Hence $p_{a}(C)=0$ and $C D=1$. Since $D \prec C+D$, by the maximality of $D, \mathcal{O}_{C+D}(-Z)$ is nef but cannot be numerically trivial. This implies $C Z<0$. Note also that $D$ has a non-multiple component meeting $C$.

We call $D$ as in the above lemma the Tyurina component of $Z$. Since $Z_{\min }$ is also the minimal model of $D$, an obvious induction gives us the longest sequence of curves

$$
0 \prec D_{m} \prec D_{m-1} \prec \cdots \prec D_{2} \prec D_{1}=Z
$$


such that $D_{i+1}$ is the Tyurina component of $D_{i}$ for $1 \leq i \leq m-1$. We call it the Yau sequence for $Z$ according to [9]. Note that $Z_{\text {min }} \preceq D_{m}$ and $D_{m} Z_{\text {min }}<0$ hold. The case $Z_{\min } Z<0$, which was excluded from the above consideration, corresponds to $m=1$. Since the Yau sequence is uniquely determined, its length $m$ is a numerical invariant of $(V, o)$. We put

$$
Y=\sum_{i=1}^{m} D_{i}
$$

and call it the Yau cycle.

Lemma 1.2. Consider the Yau sequence for $Z$ as in (1.1). Then the following hold.

(1) If $m \geq 3$, then $\operatorname{Supp}\left(D_{i}-D_{j}\right) \cap \operatorname{Supp}\left(D_{k}\right)=\emptyset$ for $i<j<k$.

(2) Choose an index $\nu$ with $1 \leq \nu \leq m$ and put $Y_{\nu}=\sum_{i=\nu}^{m} D_{i}$. Then $-Y_{\nu}$ is nef on $\operatorname{Supp}\left(D_{\nu}\right)$. In particular, $-Y$ is nef on $\pi^{-1}(o)$.

(3) $D_{i}^{2} \leq D_{i+1}^{2}$ for $1 \leq i<m$.

Proof. Assume $m \geq 3$ and take three indices $i, j, k$ with $i<j<k$. Then $\mathcal{O}_{D_{k}}\left(-\left(D_{i}-D_{j}\right)\right)$ is numerically trivial. Since $D_{k}$ is chain-connected, we have either $D_{k} \preceq D_{i}-D_{j}$ or $\operatorname{Supp}\left(D_{i}-D_{j}\right) \cap \operatorname{Supp}\left(D_{k}\right)=\emptyset$. Assume the first alternative happens. Then $D_{j}+D_{k} \preceq D_{i}$ and we get $p_{a}\left(D_{i}\right)=h^{1}\left(D_{i}, \mathcal{O}\right) \geq h^{1}\left(D_{j}+D_{k}, \mathcal{O}\right) \geq$ $p_{a}\left(D_{j}+D_{k}\right)=p_{a}\left(D_{j}\right)+p_{a}\left(D_{k}\right)-1$. When $p_{f}(V, o)>1$, this immediately leads us to a contradiction, since $p_{a}\left(D_{i}\right)=p_{a}\left(D_{j}\right)=p_{a}\left(D_{k}\right)>1$. So, we may assume that $p_{f}(V, o)=1$. Then we have $p_{a}\left(D_{i}\right)=p_{a}\left(D_{j}+D_{k}\right)=1$. Recall that, for a chainconnected curve $E$ with $p_{a}(E)>0$, any subcurve $E^{\prime} \preceq E$ satisfying $p_{a}\left(E^{\prime}\right)=p_{a}(E)$ is also chain-connected $([3, \S 3])$. Since $D_{i}$ is chain-connected, $D_{j}+D_{k}$ must be chainconnected, too. However, it is not the case, because $\mathcal{O}_{D_{k}}\left(-D_{j}\right)$ is numerically trivial. In sum, we cannot have $D_{j}+D_{k} \preceq D_{i}$. Hence (1).

We show (2). It suffices to show that $-Y$ is nef on $\operatorname{Supp}(Z)$. Take any irreducible component $C \preceq Y$ and let $i$ be the biggest index such that $C \preceq D_{i}$. Since $D_{i}$ is the fundamental cycle on its support, we have $C D_{i} \leq 0$. Furthermore, we have $C D_{j}=0$ for any $j$ satisfying either $j<i$ or $j>i+1$ by (1). So, $C Y=C D_{i}+C D_{i+1}$. If $C D_{i+1}=0$, then $C Y \leq 0$. If $C D_{i+1}>0$, then we also have $C Y \leq 0$, since Lemma 1.1 (3) implies that $C D_{i+1}=1$ and $C D_{i}<0$. Hence $-Y$ is nef. It follows $0 \geq\left(D_{i}-D_{i+1}\right) Y=\left(D_{i}-D_{i+1}\right)\left(D_{i}+D_{i+1}\right)=D_{i}^{2}-D_{i+1}^{2}$. This gives (3).

Lemma 1.3. Let the notation be as in the previous lemma. For a subcurve $\Delta \prec Y$, the following three conditions are equivalent.

(1) $-(Y-\Delta)$ is nef on $\operatorname{Supp}(\Delta)$.

(2) $-(Y-\Delta)$ is nef on $\pi^{-1}(o)$.

(3) $\Delta=Y_{\nu}$ for some $\nu, 1<\nu \leq m$.

Proof. Let $\Delta$ be a proper subcurve of $Y$. Assume that (1) holds. If $B$ is a component with $B \npreceq \Delta$, then $B \Delta \geq 0$ and $B Y \leq 0$. Hence $-B(Y-\Delta) \geq 0$. So, (2) holds. We next assume (2). Since $-(Y-\Delta)$ is nef on the chain-connected curve $Z=$ $D_{1}$ and $\operatorname{Supp}\left(D_{1}\right) \cap \operatorname{Supp}(Y-\Delta) \neq \emptyset$, we get $D_{1} \preceq Y-\Delta$, that is, $\Delta \preceq Y-D_{1}=Y_{2}$. If $\Delta \neq Y_{2}$, then, since $\mathcal{O}_{D_{2}}\left(-D_{1}\right)$ is numerically trivial, $-\left(Y_{2}-\Delta\right)=-(Y-\Delta)+D_{1}$ is nef on the chain-connected curve $D_{2}$ and $\operatorname{Supp}\left(D_{2}\right) \cap \operatorname{Supp}\left(Y_{2}-\Delta\right) \neq \emptyset$, we get $D_{2} \preceq Y_{2}-\Delta$, or equivalently, $\Delta \preceq Y_{2}-D_{2}=Y_{3}$. Now, we can show that (3) holds inductively. Clearly, (3) implies (1), because $Y-Y_{\nu}$ is numerically trivial on $Y_{\nu}$. 
Lemma 1.4. Let $\pi: X \rightarrow V$ be the minimal resolution of a numerically Gorenstein, isolated surface singularity $(V, o)$ with $p_{f}(V, o)>0$. Then $Y \preceq Z_{K}$. If furthermore $K_{Y}$ is nef and $p_{f}(V, o) \geq 2$, then $2 Y \preceq Z_{K}$.

Proof. Let $Z_{K}$ be the canonical cycle. Since $\pi$ is minimal, $-Z_{K}$ is nef on $\pi^{-1}(o)$. Let $Y=\sum_{i=1}^{m} D_{i}$ be the Yau cycle as in (1.2). Then each $D_{i}$ is chain-connected and $\mathcal{O}_{D_{j}}\left(-D_{i}\right)$ is numerically trivial for $i<j$. In particular, $\mathcal{O}_{D_{i}}\left(-\left(Z_{K}-\sum_{j=1}^{i-1} D_{j}\right)\right)$ is numerically equivalent to the nef invertible sheaf $\mathcal{O}_{D_{i}}\left(-Z_{K}\right)$ of positive degree. From this, one gets $D_{i} \preceq Z_{K}-D_{1}-\cdots-D_{i-1}$. By induction, $Y \preceq Z_{K}$.

$K_{Y}$ is numerically equivalent to $-\left(Z_{K}-Y\right)$ and it is non-trivial when $p_{f}>1$. Hence, similarly as above, one can show that $Y \preceq Z_{K}-Y$ if $K_{Y}$ is nef.

Now, the formula $p_{a}(Y)-1=\sum_{i=1}^{m}\left(p_{a}\left(D_{i}\right)-1\right)+\sum_{i<j} D_{i} D_{j}$ gives us

$$
p_{a}(Y)=m\left(p_{f}-1\right)+1 \text {. }
$$

Another numerical invariant to be investigated is $h^{1}\left(Y, \mathcal{O}_{Y}\right)$. When $m=1$, we clearly have $h^{1}\left(Y, \mathcal{O}_{Y}\right)=p_{f}$. For $m \geq 2$, we have the following:

TheOREm 1.5. When $m \geq 2$, let $\gamma$ be the order of $\mathcal{O}_{D_{m}}(-Z)($ possibly $\gamma=+\infty)$. Then

$$
h^{1}\left(Y, \mathcal{O}_{Y}\right)=\left(p_{f}-1\right) m+1+\left[\frac{m-1}{\gamma}\right],
$$

where $[(m-1) / \gamma]$ denotes the integer part of $(m-1) / \gamma$. In particular, if $\pi^{-1}(o)$ is simply connected, then $h^{1}\left(Y, \mathcal{O}_{Y}\right)=m \cdot p_{f}$.

Proof. We may assume that $\mathcal{O}_{D_{m}}(-Z)$ is a torsion element of order $\gamma$ in $\operatorname{Pic}\left(D_{m}\right)$. Consider the exact sequence

$$
0 \rightarrow \mathcal{O}_{Y_{i+1}}\left(-Y+Y_{i+1}\right) \rightarrow \mathcal{O}_{Y_{i}}\left(-Y+Y_{i}\right) \rightarrow \mathcal{O}_{D_{i}}\left(-Y+Y_{i}\right) \rightarrow 0
$$

inductively for $1 \leq i \leq m-1$. We remark that $\mathcal{O}_{Y_{i}}\left(-Y+Y_{i}\right) \simeq \mathcal{O}_{Y_{i}}$ holds if and only if $\mathcal{O}_{D_{i}}\left(-Y+Y_{i}\right) \simeq \mathcal{O}_{D_{i}}$ and $H^{0}\left(Y_{i},-Y+Y_{i}\right) \rightarrow H^{0}\left(D_{i},-Y+Y_{i}\right)$ is surjective, since $\operatorname{Supp}\left(Y_{i}\right)$ is connected and $D_{i}$ is the chain-connected component of $Y_{i}$. Furthermore, we have $\mathcal{O}_{D_{i}}\left(-Y+Y_{i}\right) \simeq \mathcal{O}_{D_{i}}(-(i-1) Z)$, since $\operatorname{Supp}\left(D_{1}-D_{i-1}\right) \cap \operatorname{Supp}\left(D_{i}\right)=\emptyset$ when $i>2$, by Lemma $1.2(1)$. Note also that $\mathcal{O}_{D_{i}}(-Z)$ for $i \geq 2$ and $\mathcal{O}_{D_{m}}(-Z)$ have the same order, since we have natural isomorphisms $H^{1}\left(D_{i}, \mathcal{O}_{D_{i}}\right) \rightarrow H^{1}\left(D_{m}, \mathcal{O}_{D_{m}}\right)$ and $H^{1}\left(D_{i}, \mathbb{Z}\right) \rightarrow H^{1}\left(D_{m}, \mathbb{Z}\right)$ by the process obtaining the minimal model (cf. [6, Lemma 3.7]).

We put $a_{i}=h^{0}\left(Y_{i},-Y+Y_{i}\right)$ for $1 \leq i \leq m$ and $a_{m+1}=0$. Then the above consideration implies

$$
a_{i}-a_{i+1}= \begin{cases}1, & \text { if } \gamma \mid(i-1) \\ 0, & \text { otherwise }\end{cases}
$$

Hence $h^{0}\left(Y, \mathcal{O}_{Y}\right)=a_{1}=\sum_{i=1}^{m}\left(a_{i}-a_{i+1}\right)=[(m-1) / \gamma]+1$. Then the formula for $h^{1}\left(Y, \mathcal{O}_{Y}\right)$ follows from the Riemann-Roch theorem. $\mathrm{u}$

REMARK 1.6. All the above results are modeled on the known facts for $p_{f}=1$. Let $(V, o)$ be an elliptic numerically Gorenstein singularity and $\pi: X \rightarrow V$ the minimal resolution. It is shown in [11, Theorem 3.7] that the Yau cycle coincides with the canonical cycle. (See also Lemma 2.1 below.) Then the formula 
for $p_{g}(V, o)=h^{1}\left(Y, \mathcal{O}_{Y}\right)$ in Proposition 1.5 is due to Okuma [6], and Lemma 1.3 corresponds to Tomari-Némethi's Lemma ([7] and [5], see also [6, Proposition 2.9]). The result corresponding to Lemma 1.2, which might be overlooked in preceding researches, can be found in [3].

\section{Applications.}

2.1. Numerically Gorenstein elliptic singularities. For the convenience of the reader, we give a proof based on the CCC decomposition for the following wellknown result due to Yau [11].

Lemma 2.1. If $(V, o)$ is a numerically Gorenstein elliptic singular point and $\pi: X \rightarrow V$ is the minimal resolution, then the Yau cycle coincides with the canonical cycle on $X$.

Proof. We compare the Yau cycle $Y=\sum_{i=1}^{m} D_{i}$ with the canonical cycle $Z_{K}$. Let $Z_{K}=\Gamma_{1}+\cdots+\Gamma_{n}$ be the CCC decomposition. It is shown in [3] that $\mathcal{O}_{\Gamma_{j}}\left(-\Gamma_{i}\right)$ is numerically trivial when $i<j, p_{a}\left(\Gamma_{i}\right)=1$ for any $i, \Gamma_{1}=Z$ and $\Gamma_{n}=Z_{\min }$. Since $D_{2}$ is the Tyurina component of $D_{1}=\Gamma_{1}$, we get $\Gamma_{2} \preceq D_{2}$. Since we are working on the minimal resolution $-Z_{K}$ is nef and, hence, $-\left(Z_{K}-\Gamma_{1}\right)$ is nef on $D_{2}$. This implies that $D_{2} \preceq Z_{K}-\Gamma_{1}$ by the chain-connectivity of $D_{2}$. Then $D_{2} \preceq \Gamma_{2}$, because $\Gamma_{2}$ is the chain-connected component of $Z_{K}-\Gamma_{1}$. Hence $D_{2}=\Gamma_{2}$. Now the obvious induction shows that $n=m$ and $D_{i}=\Gamma_{i}$ for any $i$, that is, $Y=Z_{K}$.

In the situation of the above lemma, we consider two conjectures in [6]. Put $\omega_{Y}=\mathcal{O}_{Y}(\kappa)$ and consider the exact sequence

$$
0 \rightarrow \omega_{Y_{i+1}} \rightarrow \omega_{Y_{i}} \rightarrow \mathcal{O}_{D_{i}}\left(\kappa-\left(Y-Y_{i}\right)\right) \rightarrow 0
$$

for $i=1, \ldots, m-1$. Note that $\mathcal{O}_{Y}(\kappa)$ is numerically trivial. As in the proof of Proposition 1.5, we have $h^{0}\left(\omega_{Y_{i}}\right)-h^{0}\left(\omega_{Y_{i+1}}\right)=1$ if $\mathcal{O}_{D_{i}}(\kappa) \simeq \mathcal{O}_{D_{i}}((i-1) Z) ; h^{0}\left(\omega_{Y_{i}}\right)=$ $h^{0}\left(\omega_{Y_{i+1}}\right)$ otherwise. Put $\alpha:=\min \left\{1 \leq i \leq m \mid \mathcal{O}_{D_{m}}(\kappa) \simeq \mathcal{O}_{D_{m}}((i-1) Z)\right\}$. Recall that $D_{m}$ is a minimally elliptic cycle and we have $\mathcal{O}_{D_{m}} \simeq \omega_{D_{m}}=\mathcal{O}_{D_{m}}\left(\kappa-\left(Y-Y_{m}\right)\right)$, i.e., $\mathcal{O}_{D_{m}}(\kappa) \simeq \mathcal{O}_{D_{m}}((m-1) Z)$. Hence, letting $\gamma=\operatorname{ord}\left(\mathcal{O}_{D_{m}}(Z)\right)$, we get $\gamma \mid(m-\alpha)$ and $p_{g}(V, o)=h^{0}\left(\omega_{Y}\right)=1+(m-\alpha) / \gamma$ as shown in [6].

Put $\beta=\min \left(\left\{1 \leq i<m \mid \mathcal{O}_{Y_{i+1}}\left(-\left(Y-Y_{i+1}\right)\right) \simeq \mathcal{O}_{Y_{i+1}}\right\} \cup\{m\}\right)$. By Theorem 5.13 and Corollary 2.15 in [6], respectively, we have $0 \leq \beta-\alpha<\gamma$ and see that $\beta=m$ is equivalent to $\alpha=m$. It should be noticed that the index set of the Tyurina components here is $\{1, \ldots, m\}$, while it is $\{0,1, \ldots, m\}$ in [6]; so, Okuma's $\alpha, \beta$ are smaller than ours by one, although such differences are not essential.

Proposition 2.2. Let the situation be as above. If $\beta<m$, then $\gamma \mid \beta$. In particular, Conjecture 1.4 in [6] is true, that is, $\alpha$ and $\beta$ determine each other if the resolution graph and the invariant $\gamma$ are given.

Proof. We have $\mathcal{O}_{Y_{\beta+1}}\left(-\left(Y-Y_{\beta+1}\right)\right) \simeq \mathcal{O}_{Y_{\beta+1}}$. By restricting it to $D_{m}$, we get $\mathcal{O}_{D_{m}}(-\beta Z) \simeq \mathcal{O}_{D_{m}}$ by Lemma $1.2(1)$. Hence $\gamma \mid \beta$.

Okuma also conjectured in $\left[6\right.$, Conjecture 5.14] that $D_{i}$ coincides $D_{i+1}$ on $\operatorname{Supp}\left(D_{i+1}\right)$. Unfortunately, such a strong assertion does not always hold as the following simple example shows.

EXAMPLE 2.3. Let $A_{i} 1 \leq i \leq 4$ be a non-singular rational curve satisfying $A_{1}^{2}=-4, A_{2}^{2}=A_{3}^{2}=A_{4}^{2}=-2, A_{1} A_{2}=2, A_{1} A_{3}=A_{1} A_{4}=A_{2} A_{4}=0$ and $A_{2} A_{3}=A_{3} A_{4}=1$. (See, Fig. 1.) Put $\Gamma_{1}=A_{1}+2 A_{2}+2 A_{3}+A_{4}$ and $\Gamma_{2}=A_{1}+A_{2}$. 
Then $\Gamma_{1}$ is the fundamental cycle on its support, $p_{a}\left(\Gamma_{1}\right)=1$ and $\Gamma_{2}$ is the minimal model of $\Gamma_{1}$ for which $\mathcal{O}_{\Gamma_{2}}\left(-\Gamma_{1}\right)$ is numerically trivial. Furthermore, $Z_{K}=\Gamma_{1}+\Gamma_{2}$ is the canonical cycle. $\operatorname{On} \operatorname{Supp}\left(\Gamma_{2}\right), \Gamma_{1}$ is not $\Gamma_{2}$ but $A_{1}+2 A_{2}$.

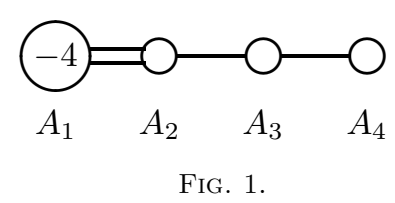

2.2. Arithmetic genus of a singularity with $p_{f}=2$. Here, we assume $p_{f}(V, o)=2$. Unlike elliptic singular points, not only the geometric genus but also the arithmetic genus can be arbitrarily big. We show that the Yau sequence gives us a natural way to compute $p_{a}(V, o)$ :

Proposition 2.4. Assume that $p_{f}(V, o)=2$. Let $k$ be the biggest positive integer such that there exists a decreasing sequence $0 \prec E_{k} \prec E_{k-1} \prec \cdots \prec E_{1}$ of chainconnected curves $E_{i}$ with support in $\pi^{-1}(o)$ satisfying

(1) $p_{a}\left(E_{i}\right)=2$ for any $i$, and

(2) $\mathcal{O}_{E_{j}}\left(-E_{i}\right)$ is numerically trivial for $i<j$.

Then $p_{a}(V, o)=p_{a}\left(\sum_{i=1}^{k} E_{i}\right)=k+1$. Furthermore, $E_{1}$ can be assumed to be $Z$.

Proof. Let $E$ be a curve whose support is in $\pi^{-1}(o)$ such that $p_{a}(E)=p_{a}(V, o)$. Let $E=E_{1}+\cdots+E_{k}$ be a CCC decomposition, where $E_{i}$ is a chain-connected curve and $\mathcal{O}_{E_{j}}\left(-E_{i}\right)$ is nef for $i<j$. We have

$$
p_{a}(E)-1=\sum_{i=1}^{k}\left(p_{a}\left(E_{i}\right)-1\right)+\sum_{i<j} E_{i} E_{j} .
$$

If $p_{a}\left(E_{i}\right)<2$ for some $i$, then $p_{a}(E)=p_{a}\left(E-E_{i}\right)+p_{a}\left(E_{i}\right)-1+E_{i}\left(E-E_{i}\right) \leq p_{a}(E-$ $\left.E_{i}\right)$. So, we can assume from the first time that $p_{a}\left(E_{i}\right)=2$ for any $i$. Then, it follows from the uniqueness of the minimal model of $Z$ that $E_{k} \preceq E_{k-1} \preceq \cdots \preceq E_{2} \preceq E_{1}$.

We have $p_{a}(E)=k+1+\sum_{i<j} E_{i} E_{j}$. If there are indices $i<j$ with $E_{i} E_{j}<0$, then $p_{a}(E)=p_{a}\left(E-E_{j}\right)+1+E_{j}\left(E-E_{j}\right) \leq p_{a}\left(E-E_{j}\right)$. This enables us to assume that $E_{i} E_{j}=0$ holds for any $i<j$. Then $E_{k} \prec E_{k-1} \prec \cdots \prec E_{2} \prec E_{1}, \mathcal{O}_{E_{j}}\left(-E_{i}\right)$ is numerically trivial for $i<j$, and $p_{a}(E)=k+1$.

We show that we can replace $E_{1}$ by $Z$. Since $E_{1}$ is chain-connected and $\mathcal{O}_{E_{1}}(-Z)$ is nef, we have $E_{1} \preceq Z$. Put $F=Z-E_{1}$ and assume $F \neq 0$. Since $\mathcal{O}_{E_{2}}\left(-E_{1}\right)=$ $\mathcal{O}_{E_{2}}(-Z+F)$ is numerically trivial and $\mathcal{O}_{E_{2}}(-Z)$ is nef, $\mathcal{O}_{E_{2}}(-F)$ is nef. Then, since $E_{2}$ is chain-connected, we have either $E_{2} \preceq F$ or $\operatorname{Supp}\left(E_{2}\right) \cap \operatorname{Supp}(F)=\emptyset$. If $E_{2} \preceq F$ is the case, then $E_{1}+E_{2} \preceq Z$ and it would follow $p_{a}\left(E_{1}+E_{2}\right) \leq h^{1}\left(E_{1}+\right.$ $\left.E_{2}, \mathcal{O}_{E_{1}+E_{2}}\right) \leq h^{1}\left(Z, \mathcal{O}_{Z}\right)=p_{a}(Z)$. This is impossible, because $p_{a}(Z)=2$ and $p_{a}\left(E_{1}+E_{2}\right)=p_{a}\left(E_{1}\right)+p_{a}\left(E_{2}\right)-1+E_{1} E_{2}=3>2$. Hence $\operatorname{Supp}\left(E_{2}\right) \cap \operatorname{Supp}(F)=\emptyset$. In particular, we see that $\mathcal{O}_{E_{2}}(-Z)$ is also numerically trivial. $\square$

It is clear that the longest sequence as in the above proposition can be realized by the Yau sequence. Hence, we get:

COROllary 2.5. Let $(V, o)$ be a normal 2-dimensional singular point with $p_{f}(V, o)=2$. Then $p_{a}(V, o)=p_{a}(Y)=m+1$ holds, where $Y$ denotes the Yau 
cycle for $Z$ as in (1.2) and $m$ is its length. In particular, $p_{a}(V, o)=2$ holds if and only if $Z_{\min } Z<0$.

Corollary 2.6. Assume that $p_{f}(V, o)=2$ and $p_{a}(V, o)>2$. Let $D$ be the Tyurina component of $Z$. If $\left(V^{\prime}, o^{\prime}\right)$ denotes the singular point obtained by contracting $D$, then $p_{a}\left(V^{\prime}, o^{\prime}\right)=p_{a}(V, o)-1$.

We give one more remark to see that $Z_{\min }$ is numerically 1 -connected when $p_{f}=2$.

LEMMA 2.7. Let $\Delta$ be a minimal, chain-connected curve of arithmetic genus 2 . Then $\Delta$ is numerically 1-connected.

Proof. Take a proper subcurve $\Gamma$ of $\Delta$. Then $p_{a}(\Gamma)<p_{a}(\Delta)=2$ and, since $K_{\Delta}$ is nef, we have

$$
0 \leq\left.\operatorname{deg} K_{\Delta}\right|_{\Gamma}=\operatorname{deg} K_{\Gamma}+(\Delta-\Gamma) \Gamma .
$$

It follows that $(\Delta-\Gamma) \Gamma \geq 0$, where the equality sign holds only if $\left.\operatorname{deg} K_{\Delta}\right|_{\Gamma}=0$ and $p_{a}(\Gamma)=1$. Assume that $(\Delta-\Gamma) \Gamma=0$ and put $\Gamma^{\prime}=\Delta-\Gamma$. Then we also have $\left.\operatorname{deg} K_{\Delta}\right|_{\Gamma^{\prime}}=0$ and $p_{a}\left(\Gamma^{\prime}\right)=1$. This is impossible, since $2=\operatorname{deg} K_{\Delta}=\left.\operatorname{deg} K_{\Delta}\right|_{\Gamma}+$ $\left.\operatorname{deg} K_{\Delta}\right|_{\Gamma^{\prime}}=0+0=0$. Therefore, $(\Delta-\Gamma) \Gamma \geq 1$, that is, $\Delta$ is numerically 1-connected. 口

3. Singularities of degree one. Let $\pi: X \rightarrow V$ be the minimal resolution of an isolated singularity $(V, o)$ of a complex surface. Throughout the section, we denote by $Z$ the fundamental cycle on $\pi^{-1}(o)$ and assume that $Z^{2}=-1$. Since $\pi$ is the minimal resolution, we automatically have $p_{f}(V, o)>0$. Note also that $Z$ is numerically 1-connected by $Z^{2}=-1$ (see, e.g., [2, Lemma 2.1]).

3.1. Canonical cycle. Firstly, we study the Tyurina component of $Z$.

LEMMA 3.1. Let $(V, o)$ be an isolated singular point of degree one and $p_{f}(V, o)>0$. Let $Z$ be the fundamental cycle on the minimal resolution and assume that $Z \neq Z_{\text {min }}$. Then $Z_{\min } Z=0$ and $Z-D$ is a (-2)-curve, where $D$ denotes the Tyurina component of $Z$.

Proof. Since $Z^{2}=-1$, we have the unique non-multiple component $A_{1}$ with $-A_{1} Z=1$ and $\mathcal{O}_{Z-A_{1}}(-Z)$ is numerically trivial. We assume that $Z$ is not minimal and let $B \prec Z$ be a $(-1)_{Z}$-curve, i.e., $B \simeq \mathbb{P}^{1}$ and $B(Z-B)=1$. Since we are working on the minimal resolution, we get $B^{2} \leq-2$. Then $B Z \leq-1$ by $B(Z-B)=1$. This implies that $B=A_{1}$ and $B^{2}=-2$, that is, $A_{1}$ is a $(-2)$-curve. It is then clear that $D=Z-A_{1}$ is the Tyurina component of $Z$. $\square$

Note that we have $D^{2}=-1$ by $-1=Z^{2} \leq D^{2}<0$. So, $D$ has a unique irreducible component $A_{2}$ of multiplicity one satisfying $-A_{2} D=1$. Since $A_{2} Z=0$, we get $A_{1} A_{2}=1$. Therefore, by induction, Yau sequence for $Z$ is of the form

$$
0 \prec Z_{\min }=D_{m} \prec D_{m-1} \prec \cdots \prec D_{1}=Z \text {, }
$$

where $D_{i}^{2}=-1$ for any $i, \mathcal{O}_{D_{j}}\left(-D_{i}\right)$ is numerically trivial for $i<j$ and $A_{i}=D_{i}-D_{i+1}$ is a $(-2)$-curve with $-A_{i} D_{i}=1$ and $A_{i} D_{i+1}=1$ for $1 \leq i<m$. In particular, $Z-Z_{\text {min }}$ is the fundamental cycle of the rational double point of type $\left(\mathbf{A}_{m-1}\right)$ and a part of the dual graph of $Z$ is as in Fig. 2. (This fact also follows from [9, Proposition 5.2].) We denote by $A$ the unique irreducible component of $D_{m}$ such that $-A D_{m}=1$. Since $D_{m}$ is minimal, $A$ is not a $(-1)_{D_{m}}$-curve. Hence, either $p_{a}(A)>0$ or $A \simeq \mathbb{P}^{1}$, $A^{2} \leq-3$. 


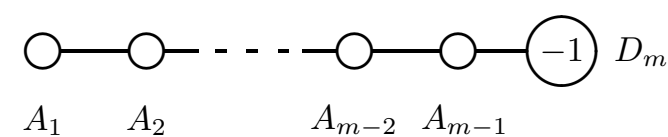

FIG. 2. Rough dual graph of $Z$

Next, we give a lower bound of $p_{a}(V, o)$ by using the Yau cycle $Y=\sum_{i=1}^{m} D_{i}$. As we shall see in Example 3.10, the bound is sharp.

Lemma 3.2. $p_{a}(V, o) \geq p_{f}\left(p_{f}-1\right) m / 2+1$ holds for an isolated surface singularity $(V, o)$ of degree one and $p_{f}(V, o)>0$.

Proof. Let $k$ be a positive integer. We have

$$
p_{a}(k Y)-1=k\left(p_{a}(Y)-1\right)+\left(\begin{array}{l}
k \\
2
\end{array}\right) Y^{2}=\left(p_{f}-1\right) m k-\frac{m}{2} k(k-1) .
$$

Then $p_{a}(k Y) \leq p_{f}\left(p_{f}-1\right) m / 2+1$, where the equality sign holds only when $k=$ $p_{f}-1, p_{f}$. In particular, we get the assertion, since $p_{a}(V, o) \geq p_{a}(k Y)$ for any $k$.

We already know that $A$ is not a (-2)-curve.

Definition 3.3. We say that $Z$ is essentially irreducible if either $Z=A$ or $Z-A$ consists of $(-2)$-curves.

We remark that, when $p_{f}=1$, the condition $Z^{2}=-1$ automatically assures that $Z$ is essentially irreducible: in fact, either $Z_{\min }=A$ or $A$ is a (-3)-curve.

Lemma 3.4. Let $(V, o)$ be a normal surface singularity with $p_{f}(V, o)>0$ and $Z^{2}=-1$. Assume that $Z$ is essentially irreducible (on the minimal resolution). Then $(V, o)$ is numerically Gorenstein with canonical cycle $\left(2 p_{f}-1\right) Y$. Furthermore, $Z_{\min }\left(=D_{m}\right)$ is numerically 2-connected and $\left|K_{Z_{\min }}\right|$ is free from base points.

Proof. We have $K_{X} A=K_{X} Z=2 p_{f}(V, o)-1>0$. We claim that $B Y=0$ for any component $B \prec Z-A$. This can be seen as follows. If $B=A_{i}$ for some $i$, $1 \leq i \leq m-1$, then we clearly have $B Y=B\left(D_{i}+D_{i+1}\right)=0$. If $B \preceq D_{m}-A$, then $\mathcal{O}_{B}\left(-D_{i}\right)$ is numerically trivial for any $1 \leq i \leq m$ and, hence, $B Y=0$. In sum, we have shown that $\left(2 p_{f}-1\right) Y$ is the canonical cycle on $\pi^{-1}(o)$, since $Z$ is essentially irreducible.

It follows from $D_{m}^{2}=-1$ that $D_{m}$ is at least numerically 1-connected (e.g., [2], Lemma 2.1). We know that $\left(2 p_{f}-1\right) D_{m}$ is the canonical cycle on $\operatorname{Supp}\left(D_{m}\right)$, because $\mathcal{O}_{D_{m}}\left(-D_{i}\right)$ is numerically trivial for $i<m$. By the adjunction formula, $K_{D_{m}}$ is numerically equivalent to $-2\left(p_{f}-1\right) D_{m}$ on $\operatorname{Supp}\left(D_{m}\right)$. If $C$ is a proper subcurve of $D_{m}$, then $\left.\operatorname{deg} K_{D_{m}}\right|_{C}=\operatorname{deg} K_{C}+C\left(D_{m}-C\right)$. It follows that $C\left(D_{m}-C\right)=$ $-2\left(p_{f}-1\right) C D_{m}+2-2 p_{a}(C)$ is even. This implies that $D_{m}$ is 2 -connected. Then it is known that $\left|K_{D_{m}}\right|$ is free from base points (see, e.g., [1, Proposition (A.7)]).

THEOREM 3.5. Let $(V, o)$ be a normal surface singularity of degree one, $p_{f}(V, o)>$ 0 and $Z$ the fundamental cycle on the minimal resolution of $(V, o)$. Assume that one of the following two conditions (1), (2) is satisfied for any curve $\Gamma$ which is the fundamental cycle on its support and satisfies $\Gamma \preceq Z, \Gamma^{2}=-1$ and $K_{\Gamma}$ nef.

(1) $\Gamma$ is essentially irreducible.

(2) The irreducible component $A_{\Gamma}$ with $A_{\Gamma} \Gamma=-1$ is a fixed component of $\left|K_{\Gamma}\right|$. 
Then $(V, o)$ is a numerically Gorenstein singularity and there exists a sequence $\left\{Z_{i}\right\}_{i=1}^{r}$ of fundamental cycles of singularities of degree one satisfying $Z=Z_{1}$ and $\mathcal{O}_{Z_{j}}\left(-Z_{i}\right)$ is numerically trivial when $i<j$, such that the canonical cycle on $\operatorname{Supp}(Z)$ can be written as

$$
Z_{K}=\sum_{i=1}^{r}\left(2 p_{i}-1\right) Y_{i}
$$

where $Y_{i}$ denotes the Yau cycle for $Z_{i}$ and $p_{i}=p_{a}\left(Z_{i}\right)(i=1, \ldots, r)$.

Proof. We do it by induction on the fundamental genus.

As already remarked, $Z$ is essentially irreducible when $p_{f}(V, o)=1$. Hence $(V, o)$ is numerically Gorenstein by Lemma 3.4.

Suppose that $p_{f}(V, o)>1$. As usual, take the Yau sequence $D_{m} \prec \cdots \prec D_{1}=Z$ for $Z$ and let $A$ be the component with $A D_{m}=-1$. Then $D_{m}$ satisfies either (1) or (2). When (1) is the case, we are done by Lemma 3.4. So, we have (2), that is, $A$ is a fixed component of $\left|K_{D_{m}}\right|$. Then it follows from [2, Theorem 1.1 and Lemma 1.4] that $A \simeq \mathbb{P}^{1}$ and $D_{m}$ decomposes as

$$
D_{m}=A+\Gamma_{1}+\cdots+\Gamma_{n-1}, A^{2}=-n,
$$

where each $\Gamma_{i}$ is the fundamental cycle on its support, $A \Gamma_{i}=-\Gamma_{i}^{2}=1$ and $\mathcal{O}_{\Gamma_{j}}\left(-\Gamma_{i}\right) \simeq \mathcal{O}_{\Gamma_{j}}$ when $i<j$. We have $p_{a}\left(\Gamma_{i}\right)>0, p_{a}\left(D_{m}\right)=\sum_{i=1}^{n-1} p_{a}\left(\Gamma_{i}\right)$ and, either $\Gamma_{j} \prec \Gamma_{i}$ or $\operatorname{Supp}\left(\Gamma_{i}\right) \cap \operatorname{Supp}\left(\Gamma_{j}\right)=\emptyset$ for $i<j$. Furthermore, if $\Gamma_{k} \prec \Gamma_{j} \prec \Gamma_{i}$, then we have $\operatorname{Supp}\left(\Gamma_{i}-\Gamma_{j}\right) \cap \operatorname{Supp}\left(\Gamma_{k}\right)=\emptyset$, because the condition $A \Gamma_{i}=A \Gamma_{j}=A \Gamma_{k}=1$ forbids $\Gamma_{k} \preceq \Gamma_{i}-\Gamma_{j}$. Note also that we have $n \geq 3$ by the minimality of $D_{m}$. Hence $p_{a}\left(\Gamma_{i}\right)$ is strictly smaller than $p_{f}(V, o)$.

After re-labeling if necessary, we may assume that $\left\{\Gamma_{i}\right\}_{i=1}^{s}, s \leq n-1$, is the set of all chain-connected components of $D_{m}-A$, i.e., maximal members in $\left\{\Gamma_{1}, \ldots, \Gamma_{n-1}\right\}$. Then $\operatorname{Supp}\left(\Gamma_{i}\right) \cap \operatorname{Supp}\left(\Gamma_{j}\right)=\emptyset$ for $i<j \leq s$. Since $p_{a}\left(\Gamma_{i}\right)<p_{a}\left(D_{m}\right)$ and $\Gamma_{i}$ is the fundamental cycle of a singularity of degree one for any $i \leq s$, the hypothesis of induction allows us to assume that $\Gamma_{i}$ contracts to a numerically Gorenstein singularity. We let $Z_{K_{i}}$ be the canonical cycle on $\operatorname{Supp}\left(\Gamma_{i}\right)$ and put $d_{i}=A Z_{K_{i}}$ for $i=1, \ldots, s$. Then $d_{i}$ is a positive integer.

Consider the integral cycle $\Xi=\left(n-2+\sum_{i=1}^{s} d_{i}\right) Y+\sum_{i=1}^{s} Z_{K_{i}}$. Recall that $\operatorname{Supp}\left(D_{1}-D_{m}\right) \cap \operatorname{Supp}\left(D_{m}-A\right)=\emptyset$ and $-Y$ is numerically trivial on $Z-A$. Using this, one easily sees that $\Xi B=-K_{X} B$ holds for any component $B$ of $Z-A$. Furthermore, since $A Y=-1$ and $A$ is a $(-n)$-curve, we get $\Xi A=2-n=-K_{X} A$ by the choice of $d_{i}$ 's. Hence, $\Xi$ gives us the canonical cycle on $\operatorname{Supp}(Z)$.

Since $Z Y=-1$ and $Z Z_{K_{i}}=0$ for any $1 \leq i \leq s$, we get $\Xi Z=-\left(n-2+\sum_{i=1}^{s} d_{i}\right)$. On the other hand, we have $K_{X} Z=2 p_{f}(V, o)-1$ by $Z^{2}=-1$. Hence the equality $n-2+\sum_{i=1}^{s} d_{i}=2 p_{f}(V, o)-1$ holds for the coefficient of $Y$ in $\Xi$.

REMARK 3.6. (1) In the above situation, similarly as in Lemma 2.4, we have

$$
p_{a}(V, o)-1 \geq \frac{1}{2} \sum_{i=1}^{r} p_{i}\left(p_{i}-1\right) m_{i},
$$

where $m_{i}$ denotes the length of the Yau sequence for $Z_{i}$.

(2) For a numerically Gorenstein singularity of degree one, $\left(2 p_{f}-1\right) Y$ is a subcurve of the canonical cycle on the minimal resolution, as we shall see in the next section. 
(3) A surface singularity of degree one is not necessarily numerically Gorenstein when $p_{f}>1$, as the following example shows. Let $C_{1}, C_{2}$ be irreducible curves with $C_{1}^{2}=$ $-4, C_{1} C_{2}=-C_{2}^{2}=3, C_{2} \simeq \mathbb{P}^{1}$. Then $C_{1}+C_{2}$ is the fundamental cycle of a singularity of degree one and $p_{f}=p_{a}\left(C_{1}\right)+2$ for which the canonical cycle $\left(2 p_{f}-1\right) C_{1}+\left(2 p_{f}-\right.$ $2 / 3) C_{2}$ is not integral.

3.2. A $p_{g}$-bound in the essentially irreducible case. We keep the notation just after Lemma 3.1.

Lemma 3.7. Let $k$ be a non-negative integer and $L$ a line bundle on $D_{i}$ numerically equivalent to $\mathcal{O}_{D_{i}}(-k Y)$. Then the restriction map $H^{0}\left(D_{i}, L\right) \rightarrow H^{0}(A, L)$ is injective.

Proof. Recall that $\operatorname{mult}_{A}\left(D_{i}\right)=1$ and consider the cohomology long exact sequence for

$$
0 \rightarrow \mathcal{O}_{D_{i}-A}(L-A) \rightarrow \mathcal{O}_{D_{i}}(L) \rightarrow \mathcal{O}_{A}(L) \rightarrow 0 .
$$

We have the decomposition $D_{i}-A=\left(D_{i}-D_{m}\right)+\left(D_{m}-A\right)$ with $\left(D_{i}-D_{m}\right) \cap\left(D_{m}-\right.$ $A)=\emptyset$. It is clear that $H^{0}\left(D_{i}-D_{m}, L-A\right)=0$, because $\mathcal{O}_{D_{i}-D_{m}}(L) \simeq \mathcal{O}_{D_{i}-D_{m}}$ and $\left(D_{i}-D_{m}\right) A=1$ when $i<m$. It follows $H^{0}\left(D_{i}-A, L-A\right) \simeq H^{0}\left(D_{m}-A, L-A\right)$. Since $\mathcal{O}_{D_{m}-A}(L-A) \equiv \mathcal{O}_{D_{m}-A}\left(D_{m}-A\right)$ and the intersection form on $D_{m}-A$ is negative definite, we get $H^{0}\left(D_{m}-A, L-A\right)=0$.

If $h^{1}\left(D_{i}, L\right)=0$, then $h^{0}\left(D_{i}, L\right)=\left.\operatorname{deg} L\right|_{D_{i}}+1-p_{f}=k+1-p_{f}$ which is not greater than $k / 2+1$ when $k \leq 2 p_{f}$. We need the following Clifford-type lemma.

Lemma 3.8. Let $L$ be as above and suppose that $h^{1}\left(D_{i}, L\right) \neq 0$. If $D_{m}-A$ supports at most exceptional sets of rational singular points, then $H^{0}\left(D_{i}, L\right) \otimes H^{0}\left(D_{i}, K_{D_{i}}-\right.$ $L) \rightarrow H^{0}\left(D_{i}, K_{D_{i}}\right)$ is non-degenerate in each factor and $h^{0}\left(D_{i}, L\right) \leq k / 2+1$.

Proof. We consider the exact sequence

$$
0 \rightarrow \mathcal{O}_{D_{i}-A}\left(K_{D_{i}-A}-L\right) \rightarrow \mathcal{O}_{D_{i}}\left(K_{D_{i}}-L\right) \rightarrow \mathcal{O}_{A}\left(K_{D_{i}}-L\right) \rightarrow 0 .
$$

By duality, $H^{0}\left(D_{i}-A, K_{D_{i}-A}-L\right)^{\vee} \simeq H^{1}\left(D_{i}-A, L\right)$ which is zero, because $D_{i}-A$ supports exceptional sets of rational singular points.

Take $s \in H^{0}\left(D_{i}, L\right)$ and $t \in H^{0}\left(D_{i}, K_{D_{i}}-L\right)$ such that $s t=0$ in $H^{0}\left(D_{i}, K_{D_{i}}\right)$. If $s \neq 0$, then $\left.s\right|_{A} \neq 0$. The same is true for $t$. Hence $s t=0$ implies that either $s$ or $t$ is zero. By Hopf's lemma, we get $h^{0}\left(D_{i}, L\right)+h^{0}\left(D_{i}, K_{D_{i}}-L\right) \leq h^{0}\left(D_{i}, K_{D_{i}}\right)+1=p_{f}+1$. By the Riemann-Roch theorem, $h^{0}\left(D_{i}, L\right)-h^{0}\left(D_{i}, K_{D_{i}}-L\right)=k+1-p_{f}$. So, $2 h^{0}\left(D_{i}, L\right) \leq k+2$.

Suppose now that $Z$ is essentially irreducible. Then, by Lemma $3.4, Z_{K}=\left(2 p_{f}-\right.$ 1) $Y$ is the canonical cycle. We shall give a bound for $p_{g}(V, o)=h^{0}\left(Z_{K}, \mathcal{O}_{Z_{K}}\right)$. When $p_{f}=1$, we have $Z_{K}=Y$ and the task has already done in [6] or Proposition 1.5 with a more accurate result. Anyway, we have $p_{g}(V, o) \leq m$ when $p_{f}=1$. So, we may assume that $p_{f} \geq 2$. By using the exact sequence

$$
0 \rightarrow \mathcal{O}_{\left(2 p_{f}-1-k\right) Y}(-k Y) \rightarrow \mathcal{O}_{\left(2 p_{f}-k\right) Y}(-(k-1) Y) \rightarrow \mathcal{O}_{Y}(-(k-1) Y) \rightarrow 0
$$

for $k=1, \ldots, 2 p_{f}-2$, one gets $h^{0}\left(Z_{K}, \mathcal{O}_{Z_{K}}\right) \leq \sum_{k=0}^{2 p_{f}-2} h^{0}(Y,-k Y)$. When $k>p_{f}-1$, we use $h^{0}(Y,-k Y)=h^{1}\left(Y, K_{Y}+k Y\right)=h^{0}\left(Y, K_{Y}+k Y\right)+\left(k-p_{f}+1\right) m$. Hence, putting $\eta=K_{X}+\left(2 p_{f}-1\right) Y$, we get

$$
p_{g}(V, o) \leq \sum_{k=0}^{p_{f}-2}\left\{h^{0}(Y,-k Y)+h^{0}(Y, \eta-k Y)\right\}+\frac{m}{2} p_{f}\left(p_{f}-1\right)+h^{0}\left(Y,-\left(p_{f}-1\right) Y\right) .
$$


If we put $L=\mathcal{O}_{Y}(-k Y)$ or $\mathcal{O}_{Y}(\eta-k Y)$, then it follows from Lemma 3.8 that $h^{0}(Y, L) \leq \sum_{i=1}^{m} h^{0}\left(D_{i}, L-\sum_{j=1}^{i-1} D_{j}\right) \leq m([k / 2]+1)$ for $0 \leq k \leq p_{f}-1$, where $[k / 2]$ denotes the integer part of $k / 2$. So,

$$
p_{g}(V, o) \leq 2 m \sum_{k=0}^{p_{f}-2}\left[\frac{k}{2}\right]+m\left(p_{f}^{2}+3 p_{f}-2\right) / 2+m\left[\frac{p_{f}-1}{2}\right]=p_{f}^{2} m .
$$

This shows the first half of the following:

THEOREM 3.9. Let $(V, o)$ be an isolated surface singularity of degree one, $Z$ the fundamental cycle on the minimal resolution. Assume that $Z$ is essentially irreducible. Then $p_{g}(V, o) \leq p_{f}^{2} m$, where $m$ denotes the length of the Yau sequence for $Z$. If $p_{g}(V, o)=p_{f}^{2} m$, then $(V, o)$ is a hypersurface double point and the maximal ideal cycle for $(V, o)$ is $Z$.

Proof. If $p_{g}(V, o)=p_{f}^{2} m$, then the above computation shows that $h^{0}\left(Y, \mathcal{O}_{Y}\right)=$ $h^{0}(Y, \eta)=m$. Hence $K_{X}$ is linearly equivalent to $-\left(2 p_{f}-1\right) Y$ and we have $\mathcal{O}_{D_{j}}\left(-D_{i}\right) \simeq \mathcal{O}_{D_{j}}$ whenever $i<j$ (see, e.g., $[3, \S 2]$ ). In particular, $(V, o)$ is Gorenstein.

Consider the cohomology long exact sequence for

$$
0 \rightarrow \mathcal{O}_{X}\left(-Z_{K}\right) \rightarrow \mathcal{O}_{X}(-Z) \rightarrow \mathcal{O}_{Z_{K}-Z}(-Z) \rightarrow 0
$$

Since $H^{1}\left(X,-Z_{K}\right)=0$ by the Kodaira-type vanishing theorem, the restriction map $H^{0}(X,-Z) \rightarrow H^{0}\left(Z_{K}-Z,-Z\right)$ is surjective. If $m=1$, then $H^{0}\left(Z_{K}-Z,-Z\right) \rightarrow$ $H^{0}(Z,-Z)$ has to be surjective and $h^{0}(Z,-Z)=1$, in order for $p_{g}(V, o)=p_{f}^{2}$ to hold. Recall that $Z$ is 2 -connected in this case by Lemma 3.4. It follows that a non-zero element in $H^{0}(Z,-Z)$ vanishes only at a non-singular point of $Z$ (see, e.g., [1, Appendix]). Assume that $m \geq 2$. Recall that $D_{1}=Z$ and consider the exact sequence

$$
0 \rightarrow H^{0}\left(Z_{K}-Z-D_{2},-Z-D_{2}\right) \rightarrow H^{0}\left(Z_{K}-Z,-Z\right) \rightarrow H^{0}\left(D_{2},-Z\right) .
$$

Since we have $p_{g}(V, o)=p_{f}^{2} m$, the restriction map $H^{0}\left(Z_{K}-Z,-Z\right) \rightarrow H^{0}\left(D_{2},-Z\right) \simeq$ $H^{0}\left(D_{2}, \mathcal{O}_{D_{2}}\right)$ has to be surjective. Since it factors through $H^{0}(Z,-Z)$, we see that $H^{0}\left(Z_{K}-Z,-Z\right) \rightarrow H^{0}(Z,-Z)$ is also non-trivial and, furthermore, the image contains an element that does not vanish on $D_{2}$. In sum, we see that $H^{0}(X,-Z) \rightarrow$ $H^{0}(Z,-Z)$ is non-trivial and there exists a section in $H^{0}(X,-Z)$ that vanishes only at a unique non-singular point $x$ of $Z\left(x \in A_{1} \backslash\left(A_{1} \cap D_{2}\right)\right)$. We have shown that $\mathcal{O}_{Z}(-Z) \simeq \mathcal{O}_{Z}(x)$.

We remark that, when $m \geq 2$, we have $h^{0}(Z,-Z)=2$ but the restriction map $H^{0}(X,-Z) \rightarrow H^{0}(Z,-Z)$ is of rank one. This can be seen as follows. Consider the exact sequence

$$
0 \rightarrow H^{0}\left(A_{1},-Z-D_{2}\right) \rightarrow H^{0}(Z,-Z) \rightarrow H^{0}\left(D_{2},-Z\right) \rightarrow 0 .
$$

Since $\mathcal{O}_{A_{1}}\left(-Z-D_{2}\right)$ and $\mathcal{O}_{D_{2}}(-Z)$ are trivial, we get $h^{0}(Z,-Z)=2$. Then, $\left|\mathcal{O}_{Z}(-Z)\right|$ is a free pencil by a result in $[3, \S 2]$. If $H^{0}(X,-Z) \rightarrow H^{0}(Z,-Z)$ were surjective, then $\mathcal{O}_{X}(-Z)$ is $\pi$-free and it would follow that $\mathfrak{m} \mathcal{O}_{X}=\mathcal{O}_{X}(-Z)$, where $\mathfrak{m}$ denotes the ideal sheaf for $o \in V$. But then $\operatorname{mult}(V, o)=-Z^{2}=1$, which is absurd.

Since $\left|\mathcal{O}_{X}(-Z)\right|$ has no fixed components, $Z$ is the maximal ideal cycle for $(V, o)$ on $X$. By using the fact that the base point $x$ of $\left|\mathcal{O}_{X}(-Z)\right|$ is a non-singular point of $Z$, it is easy to see that $\mathfrak{m} \mathcal{O}_{X} \simeq \mathfrak{m}_{x} \mathcal{O}_{X}(-Z)$ and $\operatorname{mult}(V, o)=-Z^{2}+1=2$. 
EXAMPLE 3.10. Let $(V, o)$ be a hypersurface singularity defined by $x^{a}+y^{b}+$ $z^{a b m}=0$, where $a, b, m$ are positive integers with $(a, b)=1$. We have

$$
p_{f}(V, o)=\frac{(a-1)(b-1)}{2}
$$

by Tomaru's formula [9, Theorem 4.3], $Z^{2}=-1$ and $Z_{K}=\left(2 p_{f}-1\right)\left(D_{1}+\cdots+D_{m}\right)$, where the minimal model $D_{m}$ of $Z=D_{1}$ is non-singular. One can also calculate the arithmetic genus by Tomari's formula [8, Theorem (3.8)] as:

$$
p_{a}(V, o)=\max _{r \geq 1}\left\{r\left(p_{f}-1\right)+1-\sum_{k=0}^{r-1}\left[\frac{k}{m}\right]\right\}=\frac{m}{2} p_{f}\left(p_{f}-1\right)+1 .
$$

This shows that our bound for the arithmetic genus in Lemma 3.2 is sharp.

We put $a=2, b=2 p+1$ and consider the double point defined by $x^{2}+y^{2 p+1}+$ $z^{2(2 p+1) m}=0$. Then $p_{f}=p$ and it can be checked, by using the canonical resolution for double coverings for instance, that $p_{g}(V, o)=p_{f}^{2} m$ holds. So, the bound of the geometric genus in Theorem 3.9 is also sharp.

3.3. Certain Gorenstein singularities of degree one and $p_{f}=2$. Вy a theorem of Tomari [8], we have $p_{a}(U, o) \leq p_{g}(U, o)-1$ for any Gorenstein singular point $(U, o)$. In particular, it follows that a Gorenstein surface singularity with $p_{g}=2$ is elliptic. So, the next target should be the classification of those with $p_{g}=3$. When $p_{a}(U, o)=1,(U, o)$ is an elliptic singulary by definition. When $p_{a}(U, o)=2$, we have $p_{f}(U, o)=2$ and the Yau sequence is of length one by Corollary 2.5. In view of Theorem 3.9, in which the $p_{g}$-bound is 4 when $p_{f}=2, m=1$, we have a chance to find a Gorenstein singularity of degree one satisfying $p_{f}=p_{a}=2, p_{g}=3$, among those with essentially irreducible fundamental cycle. In fact, we have:

TheOREM 3.11. Let $(V, o)$ be a Gorenstein surface singularity with $p_{f}(V, o)=2$ such that $Z^{2}=-1$ and $Z_{K}=3 Z$ hold on the minimal resolution. Then $p_{a}(V, o)=2$ and there are the following two cases, where $Z_{\mathfrak{m}}$ denotes the maximal ideal cycle.

(1) $p_{g}(V, o)=4, Z_{\mathfrak{m}}=Z, \mathfrak{m} \mathcal{O}_{X} \simeq \mathfrak{m}_{x} \mathcal{O}_{X}(-Z)$ with a non-singular point $x \in Z$, $\operatorname{mult}(V, o)=2$ and $\operatorname{embdim}(V, o)=3$.

(2) $p_{g}(V, o)=3, Z_{\mathfrak{m}}=2 Z, \mathfrak{m} \mathcal{O}_{X} \simeq \mathcal{O}_{X}(-2 Z), \operatorname{mult}(V, o)=4$ and $\operatorname{embdim}(V, o)=4$.

Proof. In the above situation, consider the cohomology long exact sequence for

$$
0 \rightarrow \mathcal{O}_{X}(-(i+1) Z) \rightarrow \mathcal{O}_{X}(-i Z) \rightarrow \mathcal{O}_{Z}(-i Z) \rightarrow 0
$$

Since $-3 Z$ is the canonical cycle, we have $H^{1}(X,-(i+1) Z)=0$ for $i \geq 2$ by the vanishing theorem. Hence $H^{0}(X,-i Z) \rightarrow H^{0}(Z,-i Z)$ is surjective when $i \geq 2$. If $H^{0}(X,-2 Z) \rightarrow H^{0}(X,-Z)$ is not surjective, then $H^{0}(Z,-Z) \neq 0$ and we get (1) of Theorem 3.11 by the proof of Theorem 3.9. So, we assume that $H^{0}(X,-2 Z) \rightarrow$ $H^{0}(X,-Z)$ is surjective. Then $2 Z \preceq Z_{\mathfrak{m}}$. Since $\left|K_{Z}\right|=\left|\mathcal{O}_{Z}(-2 Z)\right|$ is free from base points by Lemma $3.4,\left|\mathcal{O}_{X}(-2 Z)\right|$ is $\pi$-free. Hence $Z_{\mathfrak{m}}=2 Z$ and $\operatorname{mult}(V, o)=-Z_{\mathfrak{m}}^{2}=$ 4.

Claim 3.12. $H^{0}(Z,-Z)=H^{1}(Z,-Z)=0$ and $p_{g}(V, o)=3$.

Proof. To compute $p_{g}(V, o)$, we consider the cohomology long exact sequence for

$$
0 \rightarrow \mathcal{O}_{2 Z}(-Z) \rightarrow \mathcal{O}_{Z_{K}} \rightarrow \mathcal{O}_{Z} \rightarrow 0
$$


Since the restriction map $H^{0}\left(Z_{K}, \mathcal{O}_{Z_{K}}\right) \rightarrow H^{0}\left(Z, \mathcal{O}_{Z}\right)$ is surjective, we get $p_{g}(V, o)=$ $h^{0}\left(Z_{K}, \mathcal{O}_{Z_{K}}\right)=h^{0}(2 Z,-Z)+1$. Consider

$$
0 \rightarrow \mathcal{O}_{Z}(-2 Z) \rightarrow \mathcal{O}_{2 Z}(-Z) \rightarrow \mathcal{O}_{Z}(-Z) \rightarrow 0 .
$$

The restriction map $H^{0}(X,-Z) \rightarrow H^{0}(2 Z,-Z)$ is surjective by the fact that $H^{1}(X,-3 Z)=0$, while $H^{0}(X,-Z) \rightarrow H^{0}(Z,-Z)$ is zero by the assumption. It follows that $H^{0}(2 Z,-Z) \rightarrow H^{0}(Z,-Z)$ is also zero. Then $h^{0}(2 Z,-Z)=h^{0}(Z,-2 Z)=2$ and we get $p_{g}(V, o)=3$.

It remains to show that $h^{0}(Z,-Z)=0$. Since $h^{1}(2 Z,-Z)=1$, we get $h^{0}\left(2 Z, \mathcal{O}_{2 Z}\right)=1$ by the duality theorem. Then, since $H^{0}\left(2 Z, \mathcal{O}_{2 Z}\right) \rightarrow H^{0}\left(Z, \mathcal{O}_{Z}\right)$ is an isomorphism, it follows from the cohomology long exact sequence for

$$
0 \rightarrow \mathcal{O}_{Z}(-Z) \rightarrow \mathcal{O}_{2 Z} \rightarrow \mathcal{O}_{Z} \rightarrow 0
$$

that $H^{0}(Z,-Z)=H^{1}(Z,-Z)=0$.

We compute the embedding dimension. Before going in detail, we remark that $\left|\mathcal{O}_{Z}(-3 Z)\right|$ is free from base points. This can be seen as follows. If it has a base point $x$, then, by [2, Proposition 5.1], there exists a subcurve $\Delta$ of $Z$ such that $\Delta^{2}=-1$, $x$ is a non-singular point of $\Delta$ and $\mathcal{O}_{\Delta}(-3 Z) \simeq \omega_{\Delta} \otimes \mathcal{O}_{\Delta}(x)$. Since $\Delta^{2}=-1, \Delta$ is 1-connected. By $Z \Delta=0,-1$ and $\operatorname{deg} \omega_{\Delta}=2 p_{a}(\Delta)-2$, the possible case is only: $Z \Delta=-1$ and $p_{a}(\Delta)=2$. This implies that $\Delta=Z$, since $Z$ is its own minimal model. Then we get $\mathcal{O}_{Z}(-Z) \simeq \mathcal{O}_{Z}(x)$, contradicting that $H^{0}(Z,-Z)=0$.

We study the graded ring $R(Z,-Z)=\bigoplus_{i \geq 0} H^{0}(Z,-i Z)$. We have $h^{0}(Z,-2 Z)=$ 2 and $h^{0}(Z,-i Z)=i-1$ for $i \geq 3$. By the free-pencil trick, $\mu_{i}: H^{0}(Z,-i Z) \otimes$ $H^{0}(Z,-2 Z) \rightarrow H^{0}(Z,-(i+2) Z)$ is surjective for $i \geq 2, i \neq 4$. This is because $H^{1}(Z,-(i-2) Z)=0$ when $i=3$ or $i \geq 5$, while we get it by dimension count when $i=2$. Therefore, $R(Z,-Z)$ is generated in degrees at most 6 . Let $\left\{x_{0}, x_{1}\right\}$ be a basis for $H^{0}(Z,-2 Z)$. Then $H^{0}(Z,-4 Z)$ is generated by $x_{0}^{2}, x_{0} x_{1}, x_{1}^{2}$. Let $\left\{y_{0}, y_{1}\right\}$ be a basis for $H^{0}(Z,-3 Z)$. Then $H^{0}(Z,-5 Z)$ is generated by $x_{0} y_{0}, x_{0} y_{1}, x_{1} y_{0}, x_{1} y_{1}$. We consider $H^{0}(Z,-6 Z)$. Here, we have four elements $x_{0}^{j} x_{1}^{3-j}(0 \leq j \leq 3)$ which generate a subspace $V_{1}$ of codimension one. Recall that $\left|\mathcal{O}_{Z}(-3 Z)\right|$ is free from base points. By the free-pencil-trick, one can show that $\operatorname{Sym}^{2} H^{0}(Z,-3 Z) \rightarrow H^{0}(Z,-6 Z)$ is injective, and the image $V_{2}=\left\langle y_{0}^{2}, y_{0} y_{1}, y_{1}^{2}\right\rangle$ is a subspace of dimension three. We claim that $H^{0}(Z,-6 Z)=V_{1}+V_{2}$. Assume not. Then $V_{2} \subset V_{1}$ and we have three relations: $y_{0}^{2}=c_{1}(x), y_{0} y_{1}=c_{2}(x)$ and $y_{1}^{2}=c_{3}(x)$, where $c_{1}, c_{2}, c_{3}$ are cubic forms in $x_{0}, x_{1}$. It follows $y_{1} / y_{0}=c_{2}(x) / c_{1}(x)$. This implies that the morphism defined by $\left|\mathcal{O}_{Z}(-3 Z)\right|$ is the composite of the morphism defined by $\left|\mathcal{O}_{Z}(-2 Z)\right|$ and the morphism $\mathbb{P}^{1} \rightarrow \mathbb{P}^{1}$ given by $c_{2} / c_{1}$, which is impossible, because $-3 Z^{2}=3$ and $-2 Z^{2}=2$. Therefore, $V_{2} \not \subset V_{1}$. For the same reasoning, we may assume that $y_{0}^{2}, y_{1}^{2} \in V_{1}$ and $y_{0} y_{1} \notin V_{1}$. Now, we have two relations: $y_{0}^{2}=\varphi_{0}\left(x_{0}, x_{1}\right), y_{1}^{2}=\varphi_{1}\left(x_{0}, x_{1}\right)$, where $\varphi_{0}, \varphi_{1}$ are cubic forms. It is not hard to confirm that there are no further relations in $R(Z,-Z)$. Therefore, $R(Z,-Z) \simeq \mathbb{C}\left[X_{0}, X_{1}, Y_{0}, Y_{1}\right] /\left(Y_{0}^{2}-\varphi_{0}\left(X_{0}, X_{1}\right), Y_{1}^{2}-\varphi_{1}\left(X_{0}, X_{1}\right)\right)$ as graded $\mathbb{C}$-algebras, where $\operatorname{deg} X_{0}=\operatorname{deg} X_{1}=2$ and $\operatorname{deg} Y_{0}=\operatorname{deg} Y_{1}=3$.

Let $\bar{x}_{i}$ and $\bar{y}_{i}(i=0,1)$ be preimages of $x_{i}$ in $H^{0}(X,-2 Z)$ and $y_{i}$ in $H^{0}(X,-3 Z)$, respectively. Then $\bar{y}_{0}, \bar{y}_{1}$ generate $H^{0}(X,-3 Z) / H^{0}\left(X, \mathfrak{m}^{2} \mathcal{O}_{X}\right)$. Hence

$$
\operatorname{dim} \mathfrak{m} / \mathfrak{m}^{2}=\operatorname{dim} \frac{H^{0}\left(X, \mathfrak{m} \mathcal{O}_{X}\right)}{H^{0}\left(X, \mathfrak{m}^{2} \mathcal{O}_{X}\right)}=\operatorname{dim} \frac{H^{0}(X,-2 Z)}{H^{0}(X,-3 Z)}+2=h^{0}(Z,-2 Z)+2
$$

and we get $\operatorname{embdim}(V, o)=4$ as wished. 
The complete intersection singularity defined by $y^{2}=w^{3}+x^{3}, z^{2}=w^{3}-x^{3}$ in $\mathbb{C}^{4}$ (with coordinates $w, x, y, z)$ serves an example, as the above description of $R(Z,-Z)$ shows.

4. A remark on the canonical cycle. Let $\pi: X \rightarrow V$ be the minimal resolution of a numerically Gorenstein surface singularity $(V, o)$. Let $Z_{K}$ be the canonical cycle and $Z_{K}=\Gamma_{1}+\cdots+\Gamma_{n}$ a CCC decomposition, that is, each $\Gamma_{i}$ is a maximal chain-connected subcurve of $Z_{K}-\sum_{j<i} \Gamma_{j}$. When $p_{f}(V, o)>0$, we showed in [3] the following:

- $\Gamma_{1}=Z$ is the fundamental cycle and, if $n \geq 2$,

- $\Gamma_{2}=\operatorname{gcd}\left(\Gamma_{1}, Z_{K}-\Gamma_{1}\right), p_{a}\left(\Gamma_{2}\right)=p_{f}(V, o)$ and $\operatorname{Supp}\left(\Gamma_{1}-\Gamma_{2}\right) \cap \operatorname{Supp}\left(Z_{K}-\right.$ $\left.\Gamma_{1}-\Gamma_{2}\right)=\emptyset$

- $p_{a}\left(\Gamma_{i}\right)>0$ and $\Gamma_{i} \preceq \Gamma_{2}$ for any $i \geq 3$,

- for $i<j$, either $\Gamma_{j} \preceq \Gamma_{i}$ or $\operatorname{Supp}\left(\Gamma_{i}\right) \cap \operatorname{Supp}\left(\Gamma_{j}\right)=\emptyset$,

- the dualizing sheaf of every minimal curve in $\left\{\Gamma_{i}\right\}_{i=1}^{n}$ is nef.

Lemma 4.1. Assume that $p_{f}(V, o)>1$. Then $n \geq 2$ and $2-2 p_{f}(V, o) \leq \Gamma_{1} \Gamma_{2} \leq$ -1 .

Proof. If $n=1$, then $Z_{K}=Z$ and $1=p_{a}\left(Z_{K}\right)=p_{a}(Z)=p_{f}(V, o)>1$, a contradiction. Hence $n \geq 2$. We have $2 p_{a}\left(\Gamma_{1}\right)-2=\Gamma_{1}\left(K_{X}+\Gamma_{1}\right)=-\Gamma_{1}\left(Z_{K}-\Gamma_{1}\right)$. This implies that there exists an index $i \geq 2$ with $-\Gamma_{1} \Gamma_{i}>0$, if $p_{a}\left(\Gamma_{1}\right)>1$. Since $\Gamma_{i} \preceq \Gamma_{2}$ for $i \geq 2$ and $-\Gamma_{1}$ is nef, we get $-\Gamma_{1} \Gamma_{2}>0$. We have $\Gamma_{1} \Gamma_{2} \geq \Gamma_{1}\left(Z_{K}-\Gamma_{1}\right)=$ $2-2 p_{f}$.

In fact, when $p_{f}(V, o)>0$, we have $n=1$ if and only if $(V, o)$ is a minimally elliptic singularity [4].

Lemma 4.2. Assume that $i<j, \Gamma_{j} \preceq \Gamma_{i}$ and $p_{a}\left(\Gamma_{i}\right)=p_{a}\left(\Gamma_{j}\right)$. Then $\Gamma_{i}^{2} \leq \Gamma_{j}^{2}$ with equality holding only when, either $\Gamma_{i}=\Gamma_{j}$ or $\Gamma_{i}-\Gamma_{j}$ consists of (-2)-curves.

Proof. We have $2 p_{a}\left(\Gamma_{i}\right)-2=-Z_{K} \Gamma_{i}+\Gamma_{i}^{2}$. Hence $\Gamma_{j}^{2}-\Gamma_{i}^{2}=2\left(p_{a}\left(\Gamma_{j}\right)-p_{a}\left(\Gamma_{i}\right)\right)-$ $\left.Z_{K}\left(\Gamma_{i}-\Gamma_{j}\right)=-Z_{K}\left(\Gamma_{i}-\Gamma_{j}\right)\right) \geq 0$, since $-Z_{K} \equiv K_{X}$ is nef. $\square$

In particular, we have $\Gamma_{1}^{2} \leq \Gamma_{2}^{2}$.

Lemma 4.3. Assume that $\Gamma_{i+1} \preceq \Gamma_{i}$ and $\mathcal{O}_{\Gamma_{i}-\Gamma_{i+1}}\left(-\sum_{j<i} \Gamma_{j}\right)$ is numerically trivial. Then the following hold.

(1) $\Gamma_{i+1}=\operatorname{gcd}\left(\Gamma_{i}, Z_{K}-\sum_{j \leq i} \Gamma_{j}\right), p_{a}\left(\Gamma_{i+1}\right)=p_{a}\left(\Gamma_{i}\right)$ and $\operatorname{Supp}\left(\Gamma_{i}-\Gamma_{i+1}\right) \cap \operatorname{Supp}\left(Z_{K}-\right.$ $\left.\sum_{j \leq i+1} \Gamma_{j}\right)=\emptyset$.

(2) $\Gamma_{i}^{2} \leq \Gamma_{i+1}^{2}$. Furthermore, $\Gamma_{i+1}=\Gamma_{i}$ holds if and only if $\Gamma_{i}\left(\Gamma_{i}-\Gamma_{i+1}\right)=0$.

Proof. (1): Put $G=\operatorname{gcd}\left(\Gamma_{i}, Z_{K}-\sum_{j \leq i} \Gamma_{j}\right)$. Then, since $\Gamma_{i+1} \preceq G \preceq \Gamma_{i}$,

$$
\begin{aligned}
2 p_{a}(G)-2 & =-G\left(Z_{K}-G\right) \\
& =-\Gamma_{i}\left(Z_{K}-\Gamma_{i}\right)+\left(\Gamma_{i}-G\right)\left(Z_{K}-G-\sum_{j \leq i} \Gamma_{j}\right)+\left(\Gamma_{i}-G\right) \sum_{j<i} \Gamma_{j} \\
& =2 p_{a}\left(\Gamma_{i}\right)-2+\left(\Gamma_{i}-G\right)\left(Z_{K}-G-\sum_{j \leq i} \Gamma_{j}\right) .
\end{aligned}
$$

By the choice of $G, \Gamma_{i}-G$ has no common components with $Z_{K}-G-\sum_{j \leq i} \Gamma_{j}$. Hence $\left(\Gamma_{i}-G\right)\left(Z_{K}-G-\sum_{j \leq i} \Gamma_{j}\right) \geq 0$ and we get $p_{a}(G) \geq p_{a}\left(\Gamma_{i}\right)$. On the other hand, since $\Gamma_{i}$ is chain-connected, $p_{a}(G) \leq h^{1}\left(G, \mathcal{O}_{G}\right) \leq h^{1}\left(\Gamma_{i}, \mathcal{O}_{\Gamma_{i}}\right)=p_{a}\left(\Gamma_{i}\right)$. In sum, we get $p_{a}(G)=p_{a}\left(\Gamma_{i}\right)$ and $\operatorname{Supp}\left(\Gamma_{i}-G\right) \cap \operatorname{Supp}\left(Z_{K}-G-\sum_{j \leq i} \Gamma_{j}\right)=\emptyset$. Note that $G$ is 
chain-connected, since so is $\Gamma_{i}$ and $p_{a}(G)=p_{a}\left(\Gamma_{i}\right)>0$ (see, [3], Lemma 3.2). We have $G-\Gamma_{i+1} \preceq Z_{K}-\sum_{j \leq i+1} \Gamma_{j}$. So, $\mathcal{O}_{G-\Gamma_{i+1}}\left(-\Gamma_{i+1}\right)$ is nef. By the chain-connectivity of $G$ is chain-connected, this implies $\Gamma_{i+1}=G$.

(2): The first assertion follows from (1) and Lemma 4.5. To show the last equivalence, we only have to show the converse. Since $\mathcal{O}_{\Gamma_{i}-\Gamma_{i+1}}\left(-\sum_{j<i} \Gamma_{j}\right)$ is numerically trivial, we have $\left(\Gamma_{i}+\Gamma_{i+1}\right)\left(\Gamma_{i}-\Gamma_{i+1}\right)=Z_{K}\left(\Gamma_{i}-\Gamma_{i+1}\right)-\left(\Gamma_{i}-\Gamma_{i+1}\right) \sum_{j<i} \Gamma_{j}-$ $\left(\Gamma_{i}-\Gamma_{i+1}\right)\left(Z_{K}-\sum_{j \leq i+1} \Gamma_{j}\right)=Z_{K}\left(\Gamma_{i}-\Gamma_{i+1}\right)$ by (1). If $\Gamma_{i}\left(\Gamma_{i}-\Gamma_{i+1}\right)=0$, then $0 \geq\left(\Gamma_{i}-\Gamma_{i+1}\right)^{2}=-\left(\Gamma_{i}+\Gamma_{i+1}\right)\left(\Gamma_{i}-\Gamma_{i+1}\right)=-Z_{K}\left(\Gamma_{i}-\Gamma_{i+1}\right) \geq 0$. Hence $\left(\Gamma_{i}-\Gamma_{i+1}\right)^{2}=0$ and it follows $\Gamma_{i+1}=\Gamma_{i}$, since the intersection form is negative definite on $\pi^{-1}(o)$. $\square$

The following is useful to study the "leading term" of the canonical cycle in some cases.

ThEOREM 4.4. Assume that $p_{f}(V, o)>1$ and write $2 p_{f}-2=a b$ with two positive integers $a, b$. If there exist $b$ indices $i \geq 2$ satisfying $-\Gamma_{1} \Gamma_{i}=a$, then the following hold.

(1) $\Gamma_{i+1}=\operatorname{gcd}\left(\Gamma_{i}, Z_{K}-\sum_{j \leq i} \Gamma_{j}\right)$ and $p_{a}\left(\Gamma_{i+1}\right)=p_{f}(V, o)$ for $i \in\{1,2, \ldots, b\}$.

(2) $\Gamma_{b+1} \preceq \Gamma_{b} \preceq \cdots \preceq \Gamma_{2} \preceq \Gamma_{1}$ and $\Gamma_{1}^{2} \leq \Gamma_{2}^{2} \leq \cdots \leq \Gamma_{b+1}^{2}$.

(3) For $1 \leq i<j \leq b+1, \mathcal{O}_{\Gamma_{j}}\left(-\Gamma_{i}\right)$ is nef of degree $a$.

(4) For $1 \leq i<j<k \leq b+1, \operatorname{Supp}\left(\Gamma_{i}-\Gamma_{j}\right) \cap \operatorname{Supp}\left(\Gamma_{k}\right)=\emptyset$.

In particular, $p_{a}(\Gamma)=1$ and $Z_{K}-\Gamma$ is numerically equivalent to $-K_{X}$ on its support for $\Gamma=\sum_{i=1}^{b+1} \Gamma_{i}$.

Proof. We have $-\Gamma_{1} \Gamma_{i} \in\{a, 0\}$ for $i \geq 2$ by the choice of $a, b$, since $-\Gamma_{1}\left(Z_{K}-\right.$ $\left.\Gamma_{1}\right)=2 p_{f}-2$. If $\Gamma_{j} \preceq \Gamma_{i}$, then $-\Gamma_{1} \Gamma_{i} \geq-\Gamma_{1} \Gamma_{j}$. Since $\Gamma_{i} \preceq \Gamma_{2}$ for $i \geq 3$, we have $-\Gamma_{1} \Gamma_{2}=a$.

Let $i_{0}$ be the smallest index with $i_{0} \geq 3$ and $-\Gamma_{1} \Gamma_{i_{0}}=a$. Then $\Gamma_{i_{0}}$ is a maximal element in $\left\{\Gamma_{i}\right\}_{i=3}^{n}$. So, we can assume that $i_{0}=3$ after renumbering if necessary. Since $\mathcal{O}_{\Gamma_{2}-\Gamma_{3}}\left(-\Gamma_{1}\right)$ is numerically trivial, it follows from Lemma 4.3 that $\Gamma_{3}=\operatorname{gcd}\left(\Gamma_{2}, Z_{K}-\Gamma_{1}-\Gamma_{2}\right), p_{a}\left(\Gamma_{3}\right)=p_{a}\left(\Gamma_{2}\right), \Gamma_{2}^{2} \leq \Gamma_{3}^{2}$ and $\operatorname{Supp}\left(\Gamma_{2}-\Gamma_{3}, Z_{K}-\Gamma_{1}-\Gamma_{2}-\Gamma_{3}\right)=\emptyset$. Note that the last condition implies that $\Gamma_{1}, \Gamma_{2}$ and $\Gamma_{3}$ are linearly equivalent on $Z_{K}-\Gamma_{1}-\Gamma_{2}-\Gamma_{3}$. We claim that $\Gamma_{i} \preceq \Gamma_{3}$ for $i \geq 3$. If not, then $\Gamma_{3}$ and $\Gamma_{i}$ are disjoint. Then $\Gamma_{3}+\Gamma_{i} \preceq \Gamma_{2}$ and we get $p_{a}\left(\Gamma_{3}\right)+p_{a}\left(\Gamma_{i}\right)=h^{1}\left(\Gamma_{3}+\Gamma_{i}, \mathcal{O}\right) \leq h^{1}\left(\Gamma_{2}, \mathcal{O}_{\Gamma_{2}}\right)=p_{a}\left(\Gamma_{2}\right)$. This is impossible, since $p_{a}\left(\Gamma_{3}\right)=p_{a}\left(\Gamma_{2}\right)$ and $p_{a}\left(\Gamma_{i}\right)>0$. Therefore, $\Gamma_{i} \preceq \Gamma_{3}$ for $i \geq 3$.

Now, the assertions (1)-(4) can be shown inductively. The rest may be clear. $\square$

We apply Theorem 4.4 to two naive cases: (i) singularities of degree one and (ii) singularities of fundamental genus 2 .

THEOREM 4.5. Let $(V, o)$ be a numerically Gorenstein surface singular point with $p_{f}(V, o)>1$ and $Z^{2}=-1$. Then the canonical cycle on the minimal resolution decomposes as $Z_{K}=\left(2 p_{f}-1\right) Y+\Delta$, where $Y$ denotes the Yau cycle for $Z$ and, either $\Delta=0$ or $\Delta$ is a sum of (at most $p_{f}$ ) disjoint canonical cycles of singularities of fundamental genus $<p_{f}(V, o)$.

Proof. First, we show that $Z_{K}$ decomposes as $Z_{K}=\left(2 p_{f}-1\right)\left(\Delta_{1}+\cdots+\Delta_{r}\right)+\Delta$, where $\Delta_{1}=Z, \Delta_{i}$ for $i \geq 2$ is the fundamental cycle of a singularity of degree one with $p_{a}\left(\Delta_{i}\right)=p_{f}, \Delta_{r} \prec \cdots \prec \Delta_{1}$ and $\mathcal{O}_{\Delta_{j}}\left(-\Delta_{i}\right)$ is numrically trivial when $i<j$. To see this, let $Z_{K}=\sum_{i=1}^{n} \Gamma_{i}$ be a CCC decomposition. Then we have $\Gamma_{1}=Z$ and $0 \geq \Gamma_{1}\left(\Gamma_{1}-\Gamma_{2}\right)=-1-\Gamma_{1} \Gamma_{2}$. Since $-Z_{K} \Gamma_{1}=2 p_{f}-1$ and $\Gamma_{i} \preceq \Gamma_{2}$ for $i \geq 2$, 
we may assume that $\Gamma_{1} \Gamma_{i}=-1$ for $1 \leq i \leq 2 p_{f}-1$. It follows from Theorem 4.4 that $p_{a}\left(\Gamma_{i}\right)=p_{f}$ for $1 \leq i \leq 2 p_{f}-1$. Furthermore, we have $-1=\Gamma_{1}^{2} \leq \cdots \leq$ $\Gamma_{2 p_{f}-1}^{2}<0$. So, $\Gamma_{1}^{2}=\Gamma_{2}^{2}=\cdots=\Gamma_{2 p_{f}-1}^{2}=-1$ and Lemma 4.3, (2) implies that $Z=\Gamma_{1}=\Gamma_{2}=\cdots=\Gamma_{2 p_{f}-1}$. We put $\Delta_{1}=\Gamma_{1}$. Then $\left(2 p_{f}-1\right) \Delta_{1}$ is a subcurve of $Z_{K}$ and $-\Delta_{1}$ is numerically trivial on $Z_{K}-\left(2 p_{f}-1\right) \Delta_{1}$. If $Z_{K}=\left(2 p_{f}-1\right) \Delta_{1}$, then we stop by putting $r=1$ and $\Delta=0$. Assume that $Z_{K}-\left(2 p_{f}-1\right) \Delta_{1} \neq 0$. Then, $Z_{K}-\left(2 p_{f}-1\right) \Delta_{1}$ is the canonical cycle on its support (possibly with several connected components). We may assume that $\Gamma_{2 p_{f}}$ has the biggest arithmetic genus among the chain-connected components of $Z_{K}-\left(2 p_{f}-1\right) \Delta_{1}$. If $p_{a}\left(\Gamma_{2 p_{f}}\right)<p_{f}(V, o)$, then we put $\Delta=Z_{K}-\left(2 p_{f}-1\right) \Delta_{1}$ and stop with $r=1$. If $p_{a}\left(\Gamma_{2 p_{f}}\right)=p_{f}$, then we have $\Gamma_{2 p_{f}}^{2}=-1$, since $-1=\Gamma_{1}^{2} \leq \Gamma_{2 p_{f}}^{2}$ by Lemma 4.5. Note that $\Gamma_{2 p_{f}}$ is the fundamental cycle on its support, being a chain-connected component of the canonical cycle $Z_{K}-\left(2 p_{f}-1\right) \Delta_{1}$. As above, we can show $\Gamma_{2 p_{f}} \Gamma_{2 p_{f}+1}=-1, \Gamma_{2 p_{f}}^{2}=\cdots=\Gamma_{4 p_{f}-2}^{2}=-1$ and $\Gamma_{2 p_{f}}=$ $\cdots=\Gamma_{4 p_{f}-2}$. We put $\Delta_{2}=\Gamma_{2 p_{f}}$. Since $\mathcal{O}_{\Delta_{2}}\left(-\Delta_{1}\right)$ is numerically trivial, we have $\Delta_{2} \prec \Delta_{1}$. We know that $Z_{K}-\left(2 p_{f}-1\right)\left(\Delta_{1}+\Delta_{2}\right)$ is either 0 or the canonical cycle on its support. Now, the obvious induction shows the decomposition of $Z_{K}$ as claimed. Note that not only each but also the total of arithmetic genus of chain-connected components of $\Delta$ does not exceed $p_{f}$.

Next, we claim that $\Delta_{1}-\Delta_{i+1}$ is a $(-2)$-curve for $i<r$. Note that, for $i<j$, $\Delta_{i}-\Delta_{j}$ consists of (-2)-curves by Lemma 4.5 . ¿From $\left(\Delta_{i}-\Delta_{j}\right)^{2}=-2$, we know that $\Delta_{i}-\Delta_{j}$ is connected. We denote by $A_{i}$ the unique component of $\Delta_{i}$ with $-A_{i} \Delta_{i}=1$. Assume that $i<r$. We have $A_{i} \preceq \Delta_{i}-\Delta_{i+1}$ and already know that $A_{i}$ is a (-2)curve. Then $2 p_{f}-1=\left(2 p_{f}-1\right) A_{i}\left(\Delta_{i+1}+\cdots+\Delta_{\mu}\right)+A_{i} \Delta$ by $A_{i} Z_{K}=0$. Since $A_{i} \npreceq Z_{K}-\left(2 p_{f}-1\right)\left(\Delta_{1}+\cdots+\Delta_{i}\right)$, and $\Delta_{j} \preceq \Delta_{i+1}$ for $j>i+1$, we get $A_{i} \Delta_{i+1}=1$ and $A_{i} \cap \operatorname{Supp}\left(Z_{K}-\left(2 p_{f}-1\right)\left(\Delta_{1}+\cdots+\Delta_{i+1}\right)\right)=\emptyset$. Hence $A_{i}\left(\Delta_{i}-\Delta_{i+1}\right)=-2$. Since $\left(\Delta_{i}-\Delta_{i+1}\right)^{2}=-2$, we get $\left(\Delta_{i}-\Delta_{i+1}-A_{i}\right)^{2}=0$, which is sufficient to imply that $\Delta_{i}-\Delta_{i+1}=A_{i}$.

Finally, we show that $\Delta_{r} \prec \cdots \prec \Delta_{1}$ is the Yau sequence. Since the difference $\Delta_{i}-\Delta_{i+1}$ is a (-2)-curve, it suffices to show that $\Delta_{r}$ is the minimal model of $Z=\Delta_{1}$, by Lemma 3.1 and what we saw above. If $\Delta=0$, then $K_{\Delta_{r}}$ is nef, because $\Delta_{r}$ is the smallest chain-connected curve appearing in the CCC decomposition of $Z_{K}$. So, let $\Delta \neq 0$. We assume that $\Delta_{r}$ is not minimal and show that this eventually leads us to a contradiction. If $\Delta_{r}$ is not minimal, then $A_{r}$ is a (-2)-curve and $\Delta_{r}-A_{r}$ is also chain-connected of arithmetic genus $p_{f}$, by Lemma 3.1. Recall that $\Delta_{i}$ is numerically trivial on $\Delta_{r}-A_{r}$ for $i=1, \ldots, r$. Hence $\mathcal{O}_{\Delta_{r}-A_{r}}(-\Delta)$ is numerically equivalent to the nef invertible sheaf $\mathcal{O}_{\Delta_{r}-A_{r}}\left(-Z_{K}\right)$. Then, either $\Delta_{r}-A_{r} \preceq \Delta$ or $\operatorname{Supp}\left(\Delta_{r}-A_{r}\right) \cap \operatorname{Supp}(\Delta)=\emptyset$ by the chain-connectivity of $\Delta_{r}-A_{r}$. The first alternative is impossible, since it would imply the existence of a chain-connected component of $\Delta$ whose arithmetic genus is $p_{f}$. The last alternative is also impossible by the fact $\operatorname{Supp}(\Delta) \subseteq \operatorname{Supp}\left(\Delta_{r}\right)$. Therefore, $\Delta_{r}$ is minimal. $\square$

We add a remark that may be useful to study $\Delta$ further.

Lemma 4.6. Let the situation be as above and assume that $\Delta \neq 0$. Then $Z_{\min }$ decomposes as $Z_{\min }=\tilde{A}+\tilde{B}$, where

(1) $\tilde{A}$ is a 2-connected curve that is the fundamental cycle on its support, $A \preceq \tilde{A}$ and $\tilde{A}-A$ consists of $(-2)$-curves at most, where $A$ is the component with $A Z_{\min }=-1$,

(2) the set of all chain-connected components of $\tilde{B}$ coincides with that of $\Delta$,

(3) $\operatorname{Supp}(\tilde{A}-A) \cap \operatorname{Supp}(\tilde{B})=\emptyset$ and $\tilde{B}$ meets $A$. 
Proof. $Z_{\min }=\Delta_{r}$ and $A=A_{r}$ in the notation of the proof of Theorem 4.5. Since $-Z_{\text {min }}$ is numerically trivial on $\Delta$ and $\operatorname{Supp}(\Delta) \subseteq \operatorname{Supp}\left(Z_{\text {min }}-A\right)$, every chain connected component of $\Delta$, which is the fundamental cycle on its support since $\Delta$ is a sum of disjoint canonical cycles, is a subcurve of $Z_{\min }-A$. Since $\mathcal{O}_{Z_{\min }-A}(-\Delta)$ is nef, every chain-connected component of $Z_{\min }-A$ is either a subcurve of $\Delta$ or disjoint from $\operatorname{Supp}(\Delta)$. Let $\Gamma$ be a chain-connected component of $Z_{\min }-A$ disjoint from $\Delta$. Then $-K_{X} \Gamma=\left(2 p_{f}-1\right) Y \Gamma+\Delta \Gamma=0$ implying that $\Gamma$ consists of $(-2)$-curves. We define $\tilde{A}$ as the biggest subcurve of $Z_{\text {min }}$ whose support is the union of $A$ and all such $\Gamma$ 's, and put $\tilde{B}=Z_{\min }-\tilde{A}$. Then we have $(2)$ and (3). We have $-1=A Z_{\min }=A \tilde{A}+A \tilde{B}>A \tilde{A}$. Let $C$ be any component of $\tilde{A}, C \neq A$. Then it is a (-2)-curve that does not meet $\tilde{B}$, and we have $0=-K_{X} C=\left(2 p_{f}-1\right) Z_{\min } C+\Delta C=\left(2 p_{f}-1\right) \tilde{A} C$. Hence, $\mathcal{O}_{\tilde{A}}(-\tilde{A})$ is nef and $\tilde{A}^{2}=A \tilde{A}$. To show the 2-connectivity of $\tilde{A}$, we can assume that $\tilde{A}-A \neq 0$. Let $\tilde{A}=C_{1}+C_{2}$ be non-trivial decompotition by curves. We may assume $A \preceq C_{1}$. Then $C_{2}$ consists of $(-2)$-curves and it follows that $C_{2}^{2}$ is a negative even integer. By $0=\tilde{A} C_{2}=C_{1} C_{2}+C_{2}^{2}$, we get $C_{1} C_{2} \geq 2$. ㅁ

We give our second application of Theorem 4.4.

THEOREM 4.7. Let $Z_{K}$ be the canonical cycle on the minimal resolution $\pi: X \rightarrow$ $V$ of an isolated numerically Gorenstein surface singular point $(V, o)$ with $p_{f}(V, o)=2$. Then $Z_{K}$ decomposes as

$$
Z_{K}=\Delta_{1}+\cdots+\Delta_{r}+E
$$

where the $\Delta_{i}$ 's and $E$ are curves satisfying the following conditions.

(1) For any $i, 1 \leq i \leq r$, the CCC decomposition of $\Delta_{i}$ is one of the following types:

(a) $\Delta_{i}=\Gamma_{i, 1}+\Gamma_{i, 2}+\Gamma_{i, 3}, \Gamma_{i, 3} \preceq \Gamma_{i, 2} \preceq \Gamma_{i, 1}, \Gamma_{i, 1}^{2} \leq \Gamma_{i, 2}^{2} \leq \Gamma_{i, 3}^{2}$ and $\mathcal{O}_{\Gamma_{i, \nu}}\left(-\Gamma_{i, \mu}\right)$ is nef of degree 1 for $1 \leq \mu<\nu \leq 3$.

(b) $\Delta_{i}=\Gamma_{i, 1}+\Gamma_{i, 2}, \Gamma_{i, 2} \preceq \Gamma_{i, 1}, \Gamma_{i, 1}^{2} \leq \Gamma_{i, 2}^{2}$ and $\mathcal{O}_{\Gamma_{i, 2}}\left(-\Gamma_{i, 1}\right)$ is nef of degree 2 .

Furthermore, $p_{a}\left(\Gamma_{i, \nu}\right)=2, \Gamma_{i, 1}$ is the fundamental cycle on its support and, when $i<j, \mathcal{O}_{\Gamma_{j, \nu}}\left(-\Gamma_{i, \mu}\right)$ is numerically trivial and $\Gamma_{j, \nu} \prec \Gamma_{i, \mu}, \Gamma_{i, \mu}^{2} \leq \Gamma_{j, \nu}^{2}$ for any $\mu, \nu$.

(2) $Z_{K}-\sum_{j=1}^{i} \Delta_{j}$ is numerically equivalent to $-K_{X}$ on its support for any $i, 1 \leq$ $i \leq r$.

(3) If $E \neq 0$, then $E$ consists of at most two disjoint canonical cycles of numerically Gorenstein elliptic singular points. All the curves $\Gamma_{i, \nu}$ as in (1) are numerically trivial on $E$.

(4) If $E=0$, then $\Gamma_{r, \mu}$, where $\mu=3$ or 2 according to whether $\Delta_{r}$ is of type $(a)$ or (b) in (1), is the minimal model of the fundamental cycle $Z=\Gamma_{1,1}$ for $(V, o)$.

Proof. Let $Z_{K}=\sum_{i=1}^{n} \Gamma_{i}$ be a CCC decomposition. We have $2=2 p_{a}\left(\Gamma_{1}\right)-2=$ $-\Gamma_{1}\left(Z_{K}-\Gamma_{1}\right)=-\Gamma_{1} \sum_{i=2}^{n} \Gamma_{i}$. Since $\mathcal{O}_{\Gamma_{i}}\left(-\Gamma_{1}\right)$ is nef, we have $\Gamma_{1} \Gamma_{2}=-1,-2$ and, in any case, the hypothesis of Theorem 4.4 is satisfied.

We put $\Delta_{1}=\Gamma_{1}+\Gamma_{2}$ when $-\Gamma_{1} \Gamma_{2}=2$, and $\Delta_{1}=\Gamma_{1}+\Gamma_{2}+\Gamma_{3}$ when $-\Gamma_{1} \Gamma_{2}=1$. Then $p_{a}\left(\Delta_{1}\right)=1$ and $Z_{K}-\Delta_{1}$ is the canonical cycle on its support by Theorem 4.4. If $Z_{K}-\Delta_{1}=0$, then we stop with $r=1$ and $E=0$. Assume that $Z_{K}-\Delta_{1} \neq 0$. If any chain-connected component of $Z_{K}-\Delta_{1}$ is of arithmetic genus $<2$, then we stop with $r=1$ and $E=Z_{K}-\Delta_{1}$. Then $E$ consists of at most two disjoint canonical cycles of elliptic singularities. So, we may assume that $Z_{K}-\Delta_{1}$ is the canonical cycle of a singular point of $p_{f}=2$. Then, one can repeat the above argument to get $\Delta_{2}$ consisting of two or three $\Gamma_{i}$ 's of arithmetic genus 2 . Now, the obvious induction 
shows the assertions (1)-(3). We get (4), because $\Gamma_{r, \mu}$ is a minimal element in $\left\{\Gamma_{i}\right\}_{i=1}^{n}$ implying that $K_{\Gamma_{r, \mu}}$ is nef. $\mathrm{C}$

Unfortunately, we do not know whether the sequence $\Gamma_{r, 1} \prec \cdots \prec \Gamma_{1,1}=Z$ always forms the Yau sequence or not. We have $p_{a}(V, o) \geq r+1$ by Proposition 2.4.

EXAMPLE 4.8. Let $A_{i}(0 \leq i \leq 4)$ be non-singular projective curves with $A_{i}^{2}=$ -2. Suppose that the dual graph of $\mathcal{A}=\bigcup_{i=0}^{4} A_{i}$ is of Dynkin type $\left(\mathrm{D}_{5}\right)$ as in Figure 3. We denote by $(V, o)$ the singularity obtained by contracting $\mathcal{A}$. Then $Z=A_{0}+A_{1}+2 A_{2}+2 A_{3}+A_{4}$ is the fundamental cycle on $\mathcal{A}$ and we have $Z^{2}=-2$.

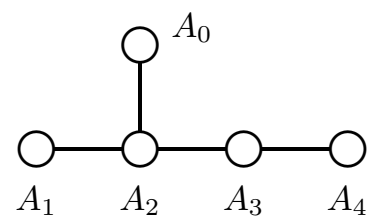

FIG. 3.

(1) This example shows that both of (a) and (b) in Theorem 4.7, (1) actually occur. Assume that $A_{0}$ is of genus two and $A_{i} \simeq \mathbb{P}^{1}$ for $1 \leq i \leq 4$. Then $p_{f}(V, o)=2$ and $Z_{K}=5 A_{0}+3 A_{1}+6 A_{2}+4 A_{3}+2 A_{4}$ is the canonical cycle. Hence $Z_{K}=\sum_{i=1}^{5} \Gamma_{i}$, where $\Gamma_{1}=\Gamma_{2}=Z, \Gamma_{3}=A_{0}+A_{1}+A_{2}, \Gamma_{4}=A_{0}+A_{2}$ and $\Gamma_{5}=A_{0}$. We have $\Gamma_{1} \Gamma_{2}=-2$ and $\Gamma_{i} \Gamma_{j}=-1$ for $3 \leq i<j \leq 5$. Put $\Delta_{1}=\Gamma_{1}+\Gamma_{2}, \Delta_{2}=\Gamma_{3}+\Gamma_{4}+\Gamma_{5}$. Then $Z_{K}=\Delta_{1}+\Delta_{2}$ is the decomposition as in Theorem 4.7, $\Delta_{2}$ is of type (a) while $\Delta_{1}$ is of type (b).

(2) Let $A_{2}$ be an elliptic curve, and $A_{i} \simeq \mathbb{P}^{1}$ for $i \neq 2$. Then $p_{f}(V, o)=2$ and the canonical cycle is $Z_{K}=3 A_{0}+3 A_{1}+6 A_{2}+4 A_{3}+2 A_{4}=\Gamma_{1}+\Gamma_{2}+\Gamma_{3}+\Gamma_{4}$, where $\Gamma_{1}=\Gamma_{2}=Z, \Gamma_{3}=A_{0}+A_{1}+A_{2}$ and $\Gamma_{4}=A_{2}$. If we put $\Delta_{1}=\Gamma_{1}+\Gamma_{2}$ and $E=\Gamma_{3}+\Gamma_{4}$, then $Z_{K}=\Delta_{1}+E$ is the decomposition as in Theorem 4.7 with $\Delta_{1}$ being of type (b) and $E$ is the canonical cycle of an elliptic singularity with fundamental cycle $\Gamma_{3}$. We get $p_{a}(V, o)=2$ from Corollary 2.5 , because $Z_{\min }=A_{0}+A_{1}+2 A_{2}+A_{3}$ and $Z_{\min } Z<0$. Note also that $\Gamma_{2} \neq Z_{\min }$. Therefore, if $E \neq 0$, the curve $\Gamma_{r, \mu}$ as in Theorem 4.7, (4) is not necessarily the minimal model of the fundamental cycle.

\section{REFERENCES}

[1] C. Ciliberto, P. Francia, and M. Mendes Lopes, Remarks on the bicanonical map for surfaces of general type, Math. Z., 224 (1997), pp. 137-166.

[2] K. Konno, On the fixed loci of the canonical systems over normal surface singularities, Asian J. Math., 12:4 (2008), pp. 449-464.

[3] K. Konno, Chain-connected component decomposition of curves on surfaces, J. Math. Soc. Japan, 62 (2010), pp. 467-486.

[4] H. B. Laufer, On minimally elliptic singularities, Amer. J. Math., 99 (1977), pp. 1257-1295.

[5] A. NÉmethi, "Weakly" elliptic Gorenstein singularities of surfaces, Invent. Math., 137 (1999), pp. 145-167.

[6] T. OKuma, Numerical Gorenstein elliptic singularities, Math. Z., 249 (2005), pp. 31-62.

[7] M. Tomari, A $p_{g}$-formula and elliptic singularities, Publ. Res. Inst. Math. Sci. Kyoto Univ., 21 (1985), pp. 297-354.

[8] M. TOMARI, Maximal-ideal-adic filtration on $R^{1} \psi_{*} \mathcal{O}_{\tilde{V}}$ for normal two-dimensional singularities, in: Complex Analytic Singularities, pp. 633-647, Adv. Stud. Pure Math., 8, NorthHolland, Amsterdam, 1987.

[9] T. Tomaru, On Gorenstein surface singularities with fundamental genus $p_{f} \geqq 2$ which satisfy some minimality conditions, Pacific J. Math., 170 (1995), pp. 271-295. 
[10] Ph. Wagreich, Elliptic singularities of surfaces, Amer. J. Math., 92 (1970), pp. 419-454.

[11] S. S.-T. YAU, On maximally elliptic singularities, Transact. AMS, 257:2 (1980), pp. 269-329. 